\title{
Gemini GMOS spectroscopy of Hell nebulae in M 33
}

\author{
C. Kehrig ${ }^{1,2}$, M. S. Oey ${ }^{2 \star \star}$, P. A. Crowther ${ }^{3}$, J. Fogel ${ }^{2}$, E. Pellegrini ${ }^{2}$, O. Schnurr ${ }^{1,3}$, \\ D. Schaerer ${ }^{4}$, P. Massey ${ }^{5, \star}$, and K. Roth ${ }^{6}$
}

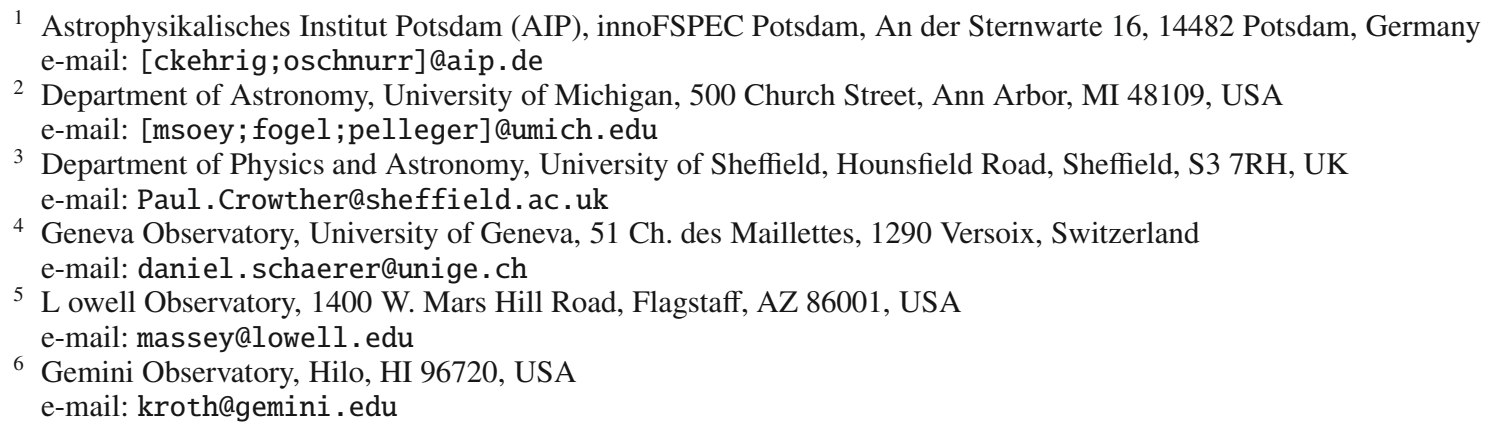

Received 29 July 2010 / Accepted 4 November 2010

\section{ABSTRACT}

\begin{abstract}
We have carried out a narrow-band survey of the Local Group galaxy, M 33, in the HeII 14686 emission line, to identify HeII nebulae in this galaxy. With spectroscopic follow-up observations, we confirm three of seven candidate objects, including identification of two new HeII nebulae, BCLMP651, HBW673. We also obtain spectra of associated ionizing stars for all the HII regions, identifying two new WN stars. We demonstrate that the ionizing source for the known HeII nebula, MA 1, is consistent with being the earlytype WN star MC8 (M33-WR14), by carrying out a combined stellar and nebular analysis of MC8 and MA 1. We were unable to identify the helium ionizing sources for HBW 673 and BCLMP 651, which do not appear to be Wolf-Rayet stars. According to the $[\mathrm{OIII}] \lambda 5007 / \mathrm{H} \beta$ vs. [NII] $\lambda 6584 / \mathrm{H} \alpha$ diagnostic diagram, excitation mechanisms apart from hot stellar continuum are needed to account for the nebular emission in HBW 673, which appears to have no stellar source at all.
\end{abstract}

Key words. stars: atmospheres - stars: Wolf-Rayet - HII regions - ISM: lines and bands - galaxies: individual: M33 galaxies: star formation

\section{Introduction}

The nebular HeII $\lambda 4686$ emission line is often observed in the integrated spectra of blue compact dwarf galaxies (BCDs; e.g., Kunth \& Sargent 1981; de Mello et al. 1998; Izotov et al. 2006a; Kehrig et al. 2008), together with other signatures of high ionization (e.g., [Ne V] and [Fe V] emission) (Thuan \& Izotov 2005). It is often seen in Wolf-Rayet (WR) galaxies, especially in lowmetallicity systems (e.g., Schaerer et al. 1999; López-Sánchez \& Esteban 2010). Locally, however, HeII emission is extremely rare in ordinary HII regions (e.g., Garnett et al. 1991; Nazé et al. 2003a), excluding planetary nebulae. What is the origin of this line? Only certain classes of WR stars, and possibly also certain $\mathrm{O}$ stars, are predicted to be hot enough to ionize nebular HeII (e.g., Kudritzki 2002; Crowther 2007), whose ionization potential $h v=54.4 \mathrm{eV}$.

In the few, known examples of HeII nebulae in the Local Group, nebular HeII emission appears to be mostly associated with individual stars (e.g., Garnett et al. 1991). For example, two nebulae (G2.4+1.4 in the Milky Way and S3 in IC 1613) are ionized by WO stars (Johnson 1975; Davidson \& Kinman 1982);

* Visiting Astronomer, Kitt Peak National Observatory, National Optical Astronomy Observatory, which is operated by the Association of Universities for Research in Astronomy (AURA) under cooperative agreement with the National Science Foundation. and four nebulae, N79, N206 in the LMC, and N76, NGC 249 in the SMC, are associated with early WN stars (Pakull 1991; Niemela et al. 1991; Testor \& Pakull 1989; Pakull 2009). The N159 F nebula in the LMC is ionized by a massive X-ray binary (LMC X-1; Pakull \& Angebault 1986), and the LMC N44 C nebula appears to be associated with a normal O7 star (Garnett et al. 2000), although it has also been suggested to be ionized by a "fossil" X-ray source (Pakull \& Motch 1989) that is no longer present.

The WR phase occurs after 3-5 Myr for high mass stars, and has relatively short durations (few $10^{5} \mathrm{yr}$ ), making WR stars vital, age-sensitive probes of high-mass star formation. Their HeII-ionizing luminosities are among the highest of any stars, reaching up to $10^{38} \mathrm{erg} \mathrm{s}^{-1}$ (e.g., Smith et al. 2002). The nebular HeII emission therefore offers a powerful probe to infer properties such as initial mass function, metallicities, and ages for distant star-forming regions. To properly understand and interpret HeII emission as a diagnostic, it is essential to establish which WR stars are responsible for nebular HeII. WR spectral energy distributions (SEDs) are notoriously difficult to model, involving complex radiation transfer in nonLTE, supersonic expanding atmospheres with important ionization stratification, inhomogeneities, and line blanketing (e.g., Crowther et al. 1995, 2002; Hamann \& Koesterke 1998). As a result, model atmospheres have varied greatly; for example, 
Schmutz \& De Marco (1999) point out very different predictions for the core effective temperature $T_{\star}$ of the prototype WN4b star, HD 50896: Schmutz (1993) predicted $T_{\star}=45000 \mathrm{~K}$, while Hamann \& Koesterke (1998) predicted $T_{\star}=100000 \mathrm{~K}$. There are especially dramatic differences in the model SEDs for the HeII edge at $228 \AA$ A. Fifteen years ago, early WC and WN stars often had been predicted to ionize $\mathrm{He}^{+}$(e.g., Schaerer 1996), whereas more recent models now show softer SEDs and only occasional $\mathrm{He}^{+}$-ionizing emission (e.g., Crowther et al. 2002a; Smith et al. 2002). The elusiveness of HeII nebulae is consistent with this latter trend. However, it contrasts with the observed large number of nebular HeII-emitting WR galaxies (Schaerer et al. 1999).

A possible explanation for nebular HeII emission in metaldeficient objects is that in such low metallicity environments, WR stars possess sufficiently weak winds that are optically thin in the $\mathrm{He}^{+}$continuum (e.g., Schmutz et al. 1992), thereby allowing the $\mathrm{He}^{+}$-ionizing radiation to escape from the stellar atmospheres. Thus, empirical determination of the stellar parameter space associated with nebular HeII photoionization will provide much-needed constraints for WR model atmospheres (e.g., Schaerer 1996; Crowther et al. 1999; Schaerer 2002).

Outside the Local Group, Brinchmann et al. (2008) analysed spectra of $570 \mathrm{WR}$ galaxies from SDSS6 and conclude that at $12+\log \mathrm{O} / \mathrm{H}<8.0$, the main sources of HeII ionizing photons appears to be $\mathrm{O}$ stars, arguing for a less dense stellar wind at these metallicities, while at higher abundances WN stars might increasingly dominate the ionization budget. De Mello et al. (1998) showed that the observed equivalent widths of nebular HeII $\lambda 4686$ emission in the BCD IZw 18 can be reproduced from an instantaneous burst model with the present WC/WO stars. They also demonstrate that there is a spatial correlation of nebular HeII $\lambda 4686$ with the position of WR stars. A similar result was reported by Kehrig et al. (2008) who found WR stars in the BCD IIZw70 by means of integral field spectroscopy. They find that the region occupied by the likely ionizing cluster is associated to the nebular emission in $4686 \AA$ indicating that the hard ionizing radiation responsible for the nebular HeII $\lambda 4686$ emission can be related to the WR stars. However, although WR and $\mathrm{O}$ stars could be responsible for most of the HeII emission in BCDs and WR galaxies (e.g., Schaerer 1996), other processes are apparently needed to explain this high ionization in a significant fraction of them (e.g., Guseva et al. 2000; Thuan \& Izotov 2005)

Models of HeII $\lambda 4686$ as a diagnostic have already been computed, both without (e.g., Schaerer 1996, 2002; Smith et al. 2002) and with supernovae remnants (SNRs; Cerviño et al. 2002). The metal-poor sensitivity is a primary motivation to develop diagnostics for relatively unevolved starbursts, and strong nebular HeII $\lambda 1640,4686$ emission is expected to be one of the best signatures of massive Population III stars (e.g., Schaerer 2002; Schaerer 2003 and references therein). Several searches for this spectral signature in high-redshift galaxies have been carried out (e.g., Dawson et al. 2004; Nagao et al. 2008; Ouchi et al. 2008, and review in Schaerer 2008).

To better understand the actual source of HeII nebular emission, we have carried out a systematic search for HeII nebulae in the Local Group galaxy, M33. The proximity of M33, together with its relatively low inclination $\left(i=53^{\circ}\right)$ and high star-formation rate, makes this an ideal target for such a study. At its distance of $840 \mathrm{kpc}$ (Freedman et al. 2001), 1" 4 pc. Moreover, M 33 has a well-determined metallicity gradient (Rosolowsky \& Simon 2008; hereafter RS08), allowing us to search for objects over a range of metallicities. For example, the case of G2.4+1.4, in the direction of the Galactic Center, suggests that HeII nebulae can also form in metal-rich environments.

The paper is organized as follows. In Sect. 2, we report narrow-band imaging to identify candidate HeII nebulae, and follow-up spectroscopic observations. The nebular properties of our HII regions are discussed in Sect. 3. In Sect. 4, we describe our sample of confirmed HeII nebulae, including a detailed analysis of the object MA 1 and its resposible ionizing star, MC8. In Sect. 5 we discuss our results. Finally, Sect. 6 summarizes the main conclusions derived from this work.

\section{Observations and data reduction}

\subsection{Narrow-band imaging}

We obtained HeII narrow-band imaging of M33 with the MOSAIC imaging camera on the 4-m Mayall Telescope at Kitt Peak National Observatory, on November 3, 2003. We used the "WRHeII" filter centered at $4690 \AA$ for emission-line imaging, and the "WR475" filter centered at $4750 \AA$ for continuum observations. Both filters have a transmission full width at halfmaximum $(F W H M)$ of about $50 \AA$. The $36^{\prime}$ MOSAIC field of view allowed us to image essentially the entire star-forming disk of M33 in two fields, North and South (Fig. 1). These fields are centered at the same positions as the $\mathrm{H} \alpha$ narrow-band images obtained by one of us (PM) as part of the NOAO Survey of Resolved Stellar Content of Local Group Galaxies, which were also obtained with the MOSAIC camera. We observed in 20-min exposures for a total of $100 \mathrm{~min}$ on the South field, and $140 \mathrm{~min}$ on the North field.

The data were reduced using the MSCRED routines in $\mathrm{IRAF}^{1}$, including bias subtraction, flat-fielding, and bad pixel correction. The MOSAIC camera also generates a low-level reflection of the pupil, which was removed with the standard tools for this purpose in the MSCRED software package. The final, combined continuum image was then registered with the corresponding line image, again using the MSCRED tools.

From the continuum-subtracted images, we visually identified 12 candidate HeII nebulae in M33. The HeII emission is faint and apparently diffuse in all cases. Seven of the objects are diffuse HII regions, mostly associated with WR stars, while five are confirmed young SNRs.

Figure 1 displays a portion $\left(0.5^{\circ} \times 0.5^{\circ}\right)$ of the $\mathrm{H} \alpha$ images obtained previously, as described above. North and South fields of M33 are shown, with the location of the 12 observed HeII nebulae identified. The HeII emission-line, continuum and continuum-subtracted images of our seven HII regions are shown in Fig. 2.

\subsection{Spectrophotometric data}

We obtained follow-up spectroscopic data of our sample objects with the Gemini Multi-Object Spectrograph (GMOS; Hook et al. 2004) at the Gemini-North observatory. We were awarded two different runs, one in service mode during semester 2006B (program GN-2006B-Q-83), and another in classical mode during the nights 2008 September 24-26 (program GN-2008B-C-5).

1 IRAF is distributed by the National Optical Astronomical Observatories, which are operated by the Association of Universities for Research in Astronomy, Inc., under cooperative agreement with the National Science Foundation. 


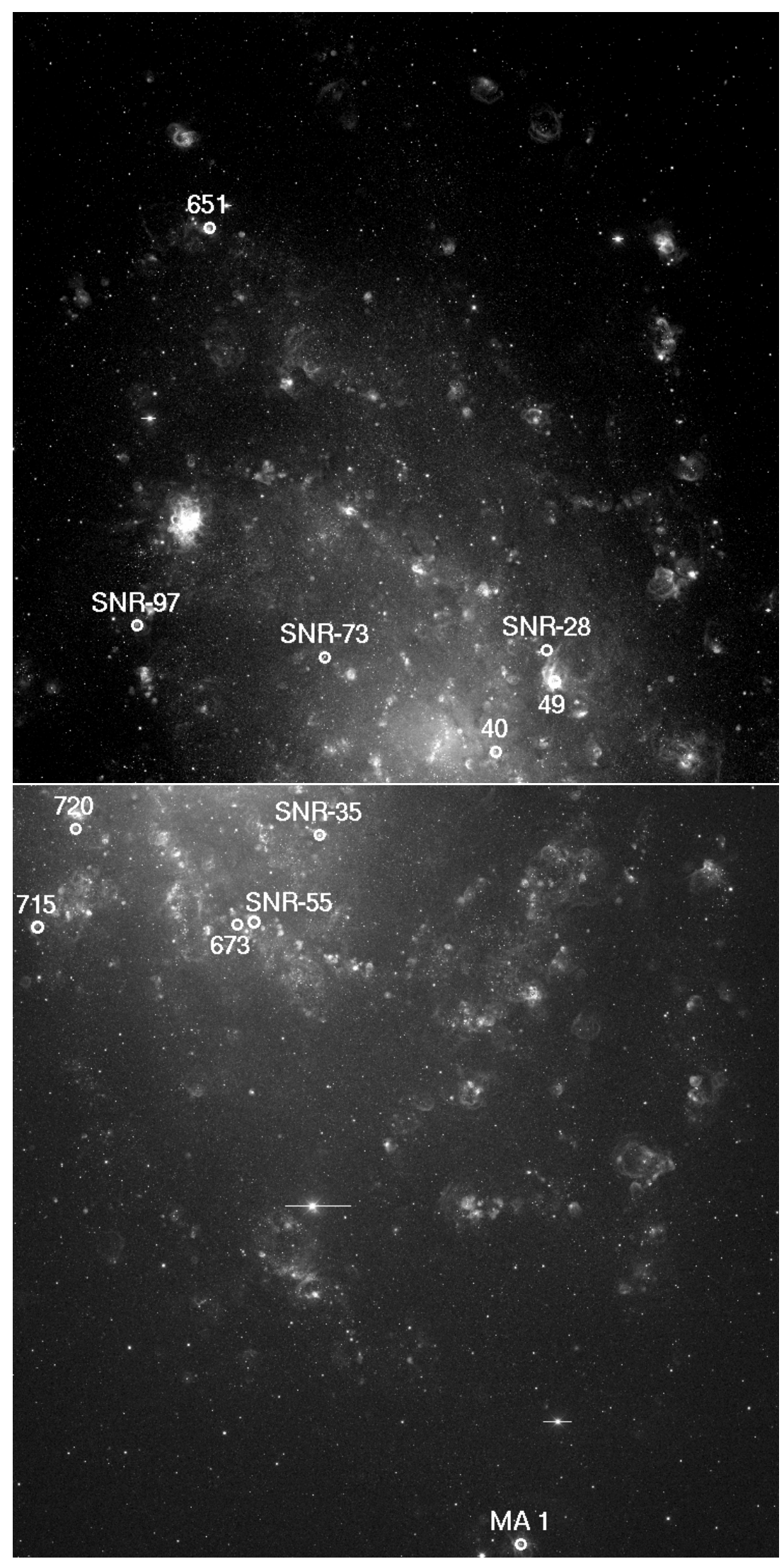

Fig. 1. Locations of the 12 candidate HeII nebulae (seven HII regions and five SNRs) superimposed on the H $\alpha$ image from the NOAO Survey of Resolved Stellar Content of Local Group Galaxies (PI: P. Massey). The labeled ID of the seven HII regions correspond to the first column of Table 1. North and South fields of M 33 are shown by the top and bottom figures, respectively. Angular size is $0.5^{\circ} \times 0.5^{\circ}$. The spatial resolution is $0.26^{\prime \prime} /$ pix. North is up and east to the left. 

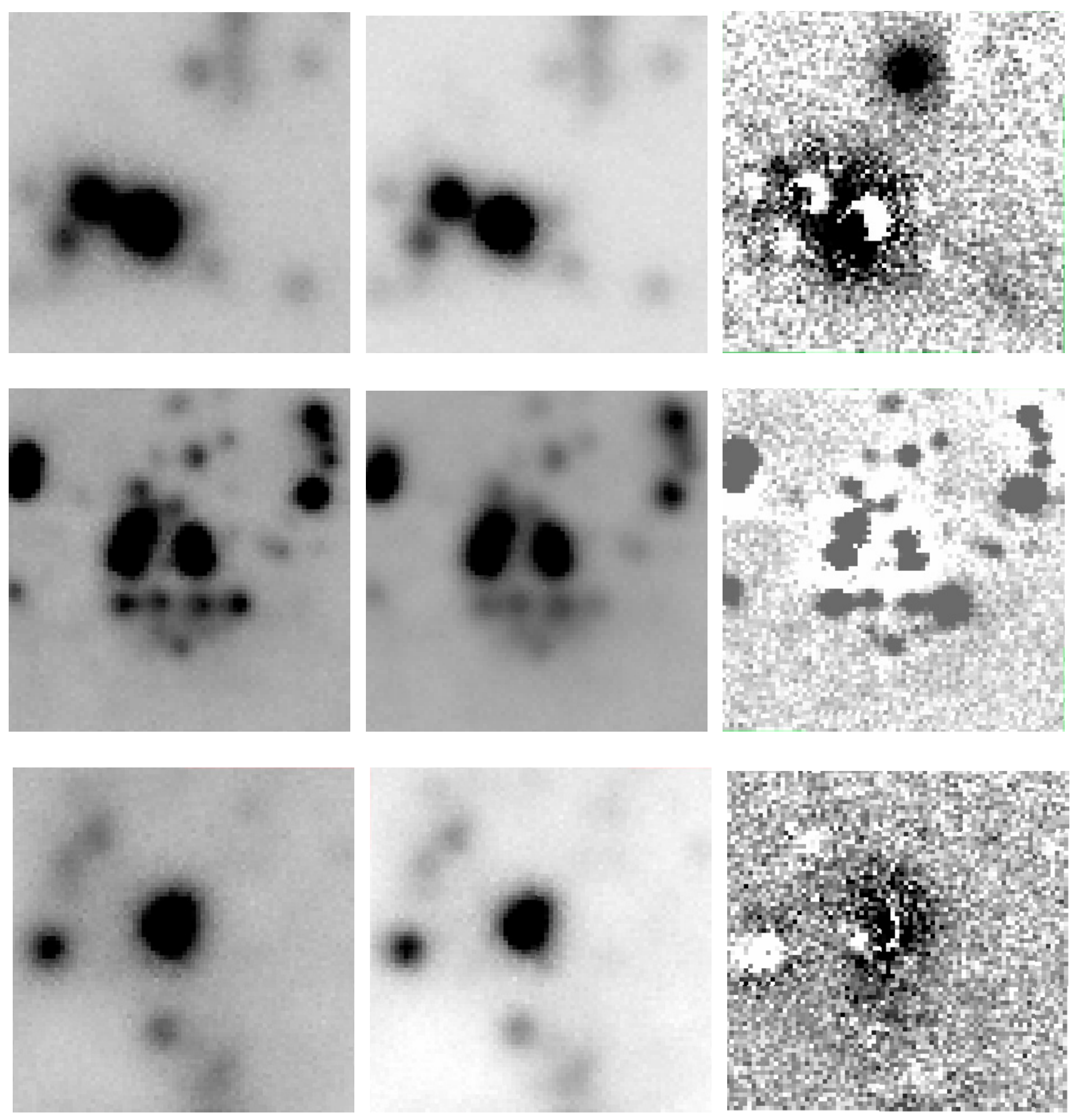

Fig. 2. HeII emission-line (left panels), continuum (middle panels) and continuum-subtracted HeII (right panels) images for our HII region sample. Top row: MA 1 - middle row: BCLMP49 - bottom row: BCLMP40. Angular size of each image is $20^{\prime \prime} \times 20^{\prime \prime}\left(\sim 80 \times 80 \mathrm{pc}^{2}\right.$ at our assumed distance of $840 \mathrm{kpc}$ ). The spatial resolution and orientation are the same as in Fig. 1 (see Sect. 2.1 for more details about the narrow-band images).

The seven target HII regions are listed in Table 1. Columns (1) and (2) show the ID for each object from the catalogs of Boulesteix et al. (1974), Hodge et al. (1999), and Mayall \& Aller (1942). Columns (3) and (4) give the object coordinates. Column (5) quotes the deprojected galactocentric distance of each nebula for our assumed distance of $840 \mathrm{kpc}$ to M 33 and an inclination angle of 56 degrees (Zaritsky et al. 1989). Column (6) gives the long-slit position angle (PA) used for each object. The last column shows the corresponding spectroscopic observing date. Figure 3 shows our finding charts for the sample, using the $\mathrm{H} \alpha$ emission-line images in Fig. 1. The associated hot stars (see Table 3) are identified, together with the slit positions for our observations (green boxes). Yellow boxes show where the nebular HeII emission falls within the slit, and represent the apertures for our extracted spectra. 

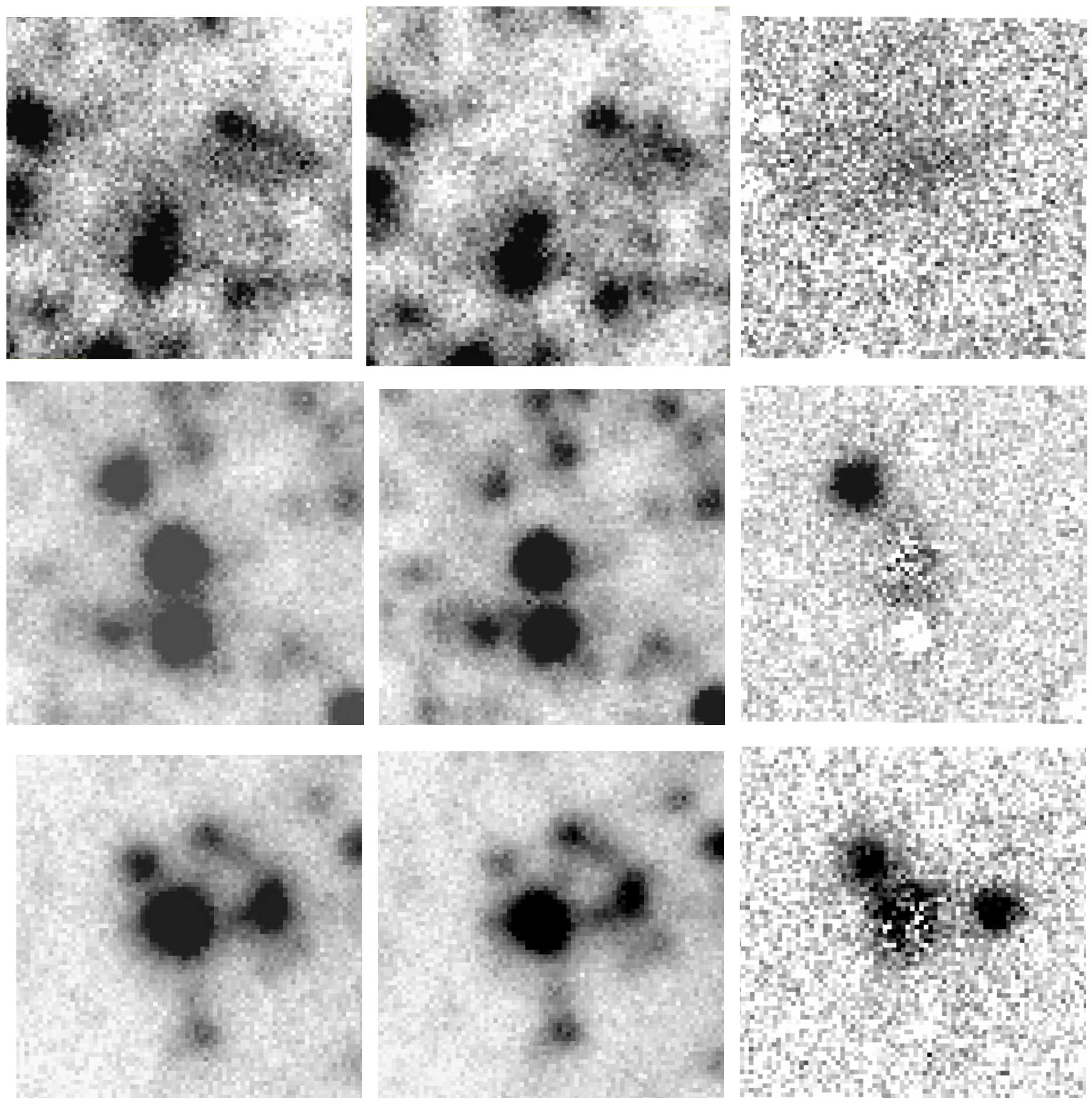

Fig. 2. continued. Top row: HBW673 - middle row: BCLMP720 - bottom row: BCLMP715.

The GMOS detector array consists of three $2048 \times$ 4608 EEV CCDs in a row. We used the B600 grating in the blue, covering a spectral range $\sim 3600-6400 \AA$ (centered at $5000 \AA$ ). On the red side, the R600 (centered at $7730 \AA$ ) was utilized, providing a spectral range from $\sim 6300$ to $9110 \AA$ A. The data were binned by a factor of 4 in the spatial dimension and by 2 in the spectral dimension, yielding $\sim 0.29^{\prime \prime} /$ pix and a spectral resolution of $\sim 3.6 \AA F W H M$ sampled at $\sim 0.9 \AA /$ pixel. The complete spectroscopic setup information is given in Table 2. We obtained spectra of both the nebulae and their associated ionizing stars, using a slit width of $0.75^{\prime \prime}$. Observations of the spectrophotometric standard stars $\mathrm{BD}+284211$ and $\mathrm{BD}+254655$ were obtained during the observing nights for flux calibration. Bias frames, dome flat-fields and copper-argon arc exposures were taken as part of the Gemini baseline calibrations.

The raw images were processed using the Gemini GMOS routines within IRAF. Biases and flat-fields were combined with the tasks GBIAS and GSFLAT, respectively. Science targets and standard stars were reduced with task GSREDUCE, which applies the overscan correction, subtracts off the bias, mosaics the three 

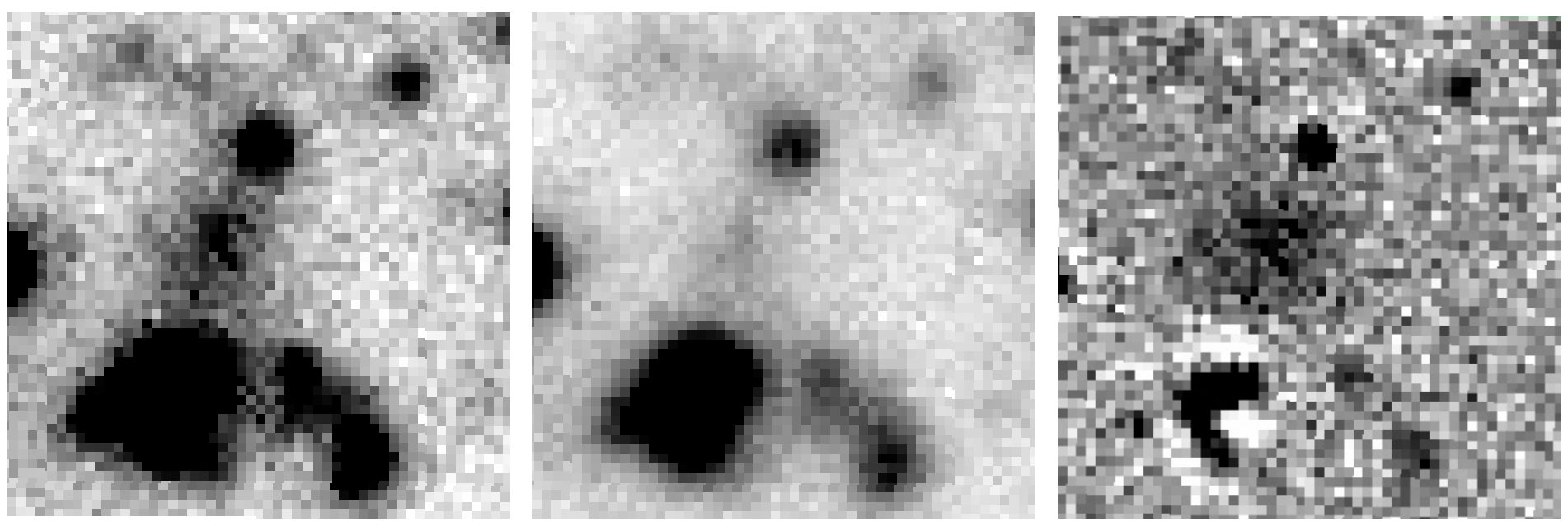

Fig. 2. continued. BCLMP651.

Table 1. The sample of our candidates for HeII-emitting HII regions.

\begin{tabular}{lcccccc}
\hline \hline Name $^{a}$ & Other designation & $\begin{array}{c}\text { RA } \\
(\mathrm{J} 2000.0)\end{array}$ & $\begin{array}{c}\text { Dec } \\
(\mathrm{J} 2000.0)\end{array}$ & $\begin{array}{c}\text { Galactocentric } \\
\text { distance }(\mathrm{kpc})\end{array}$ & $\begin{array}{c}\text { Slit PA } \\
(\text { degrees })\end{array}$ & Observing date \\
\hline MA 1 & HII-anon & 013302.2 & +301118.8 & 7.3 & 246.6 & 25 Sep. 2008 \\
BCLMP 49 & NGC 595 & 013333.5 & +304133.6 & 1.4 & 90.0 & 24 Sep. 2008 \\
BCLMP 40 & & 013343.1 & +303906.2 & 0.6 & 100.0 & 24 Sep. 2008 \\
HBW 673 & HII-z29 & 013349.5 & +303319.5 & 1.7 & 21.6 & 29 Aug. 2006 \\
BCLMP 720 & & 013416.0 & +303642.8 & 2.0 & 213.4 & 24 Sep. 2008 \\
BCLMP 715 & & 013422.3 & +303313.8 & 2.6 & 215.3 & 24 Sep. 2008 \\
BCLMP 651 & & 013429.5 & +305716.6 & 4.7 & 340.4 & 26 Sep. 2008 \\
\hline
\end{tabular}

Notes. ${ }^{(a)}$ Catalog abbreviations are the following: BCLMP, Boulesteix et al. (1974); MA, Mayall \& Aller (1942); HBW, Hodge et al. (1999) .

detectors of GMOS interpolating across the chip gaps for science data, and applies the flat field correction. The wavelength calibration was established from the arcs with the task GSWAVELENGTH giving residuals $<0.1 \AA$. The science spectra were further wavelength calibrated by applying the task GSTRANSFORM. We use the task GSEXTRACT to extract the one dimension spectra performing sky subtraction. The sensitivity function was created by using the task GSSTANDARD, and finally we flux calibrated the science images making use of the task GSCALIBRATE. The uncertainty in the instrument response fitting across the spectrum for blue and red sides is $<2 \%$.

In Table 3 we list the dominant ionizing stars for our HII regions, and their rectified, blue spectra are plotted in Fig. 4. These stellar spectra were used to estimate spectral classifications. For three of the stars in Table 3, designated as K10-nn, spectral types were not known before. We also refine spectral types provided previously by others. We base our classification of WN stars on the line ratios proposed by Smith et al. (1996) (e.g., HeII $\lambda 5411 / \mathrm{HeI} \lambda 5875 ;$ NV $\lambda 4604 / \mathrm{NIII} \lambda 4640 ;$ NIV $\lambda 4057 / \mathrm{NV}-$ III $\lambda 4604-40)$. The two WC stars in our sample were classified using the CIII $\lambda 5696 /$ CIV $\lambda 5808$ line ratio (Crowther et al. 1998). We also confirm the luminous blue variable (LBV)-like spectrum of the M 33 star J013416.07+303642.1 classified previously as a P Cyg LBV candidate by Massey et al. (2007). For three stars in our sample we are not able to give any spectral type since their spectra have low $\mathrm{S} / \mathrm{N}$ and show no obvious stellar features.

Blue and red spectra for the HII regions are displayed in Figs. 5 and 6, respectively. From these spectra, we are able to confirm nebular HeII emission in three of the seven HII regions: MA 1, BCLMP651 and HBW673, the last two being new
HeII-emitting nebulae identified for the first time. Portions of our two dimensional spectra of MA 1, BCLMP651 and HBW673 are shown in Fig. 7 from which we can see spatially extended HeII $\lambda 4686$ emission, thus confirming its nebular nature. Figure 8 presents the wavelength range 4255-4775 $\AA$ for these three nebulae, from which we can see the faint, temperature sensitive emission line [OIII] $\lambda 4363$ and the nebular HeII $\lambda 4686$.

Emission-line fluxes were measured in the 1D spectra (Figs. 5 and 6) using the IRAF task SPLOT, which integrates the line intensity from a locally fitted continuum. The derived line fluxes were computed via gaussian fitting. We carried out an iterative procedure to derive both the logarithmic reddening, $\mathrm{C}(\mathrm{H} \beta)$, and the equivalent width of the absorption in the hydrogen lines, $E W_{\text {abs }}$, which we use to correct the measured line intensities for both effects. We used the Galactic reddening law of Cardelli et al. (1989) and assumed that $E W_{\text {abs }}$ is the same for all Balmer lines (e.g., Kobulnicky \& Skillman 1996; Kobulnicky et al. 1999). We computed a linear fit to the ratio of the measuredto-theoretical Balmer decrements as a function of the aforementioned reddening law, while simultaneously varying the values for the $E W_{\text {abs }}$. This fit provides a value of $\mathrm{C}(\mathrm{H} \beta)$ for each value of $E W_{\text {abs. }}$. We then adopted the $E W_{\text {abs }}$ and $\mathrm{C}(\mathrm{H} \beta)$ values from the best fit (i.e. with minimum chi-square). The uncertainty of the fit is adopted as the error in $\mathrm{C}(\mathrm{H} \beta)$. In order to estimate how the $E W_{\text {abs }}$ correction affects our results, we compare the values of $\mathrm{C}(\mathrm{H} \beta)$ with and without the aforementioned $E W_{\text {abs }}$ correction, and we find that most are the same within the uncertainties. This indicates that, in general, the contribution of the $E W_{\text {abs }}$ in the final flux measurements is not significant. Moreover, in all 

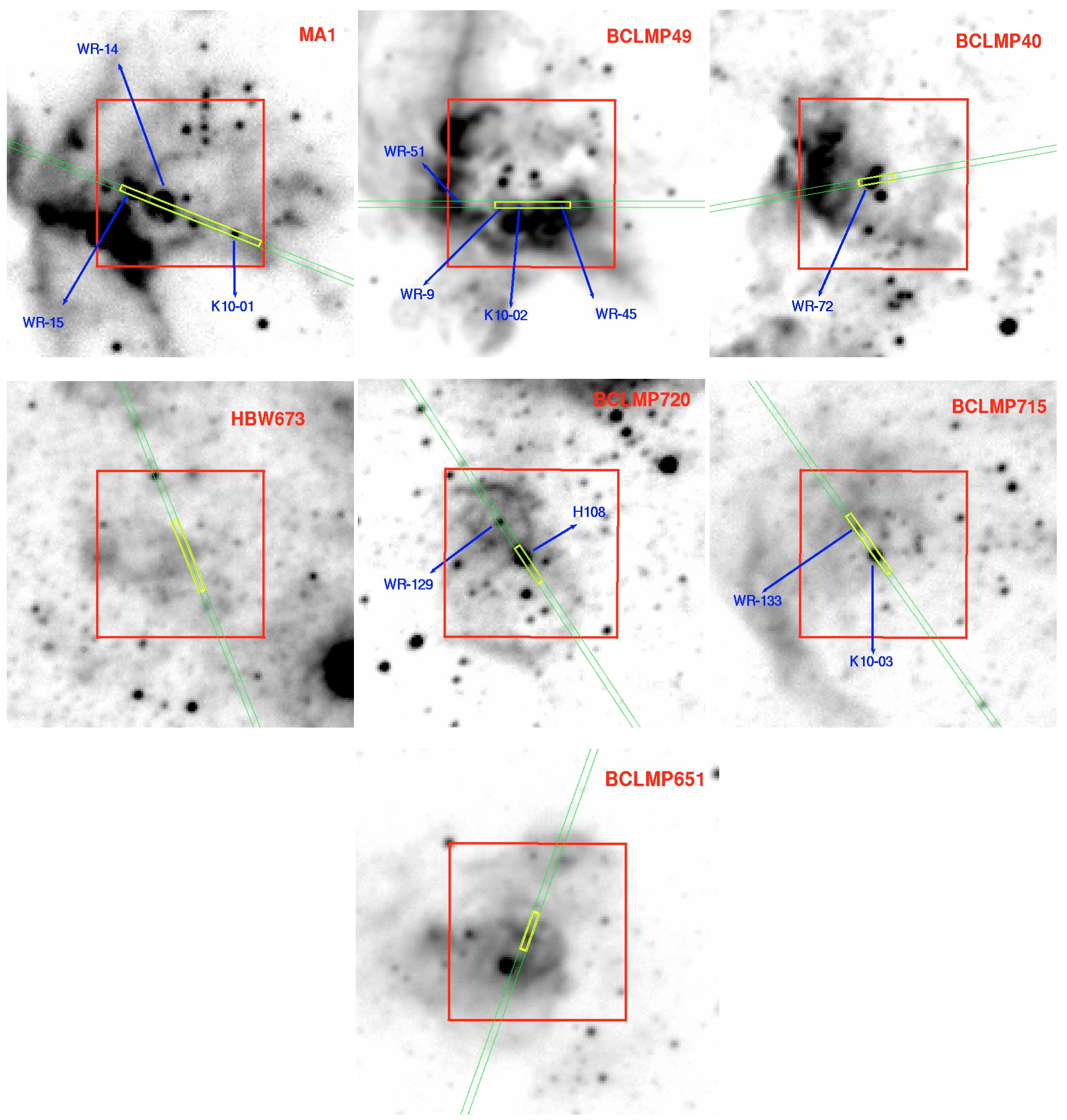

Fig. 3. Slit position (green/light grey box) for all HII regions overplotted on the $\mathrm{H} \alpha$ image from the NOAO survey of resolved stellar content of local group galaxies (PI: P. Massey). Angular size is $40^{\prime \prime} \times 40^{\prime \prime}\left(\sim 160 \times 160 \mathrm{pc}^{2}\right.$ at our assumed distance of $\left.840 \mathrm{kpc}\right)$. Yellow/light box marks where the nebular HeII emission is located within the slit. Blue arrows indicate the position and name of the associated hot stars. Red/dark grey box shows the size of the HeII images $\left(20^{\prime \prime} \times 20^{\prime \prime}\right)$ as seen in Fig. 2. The spatial resolution and orientation are the same as in Fig. 1 . The H $\alpha$ image is shown in logarithmic scale to better enhance all the morphological features of the HII regions.

cases, our estimations for the $E W_{\text {abs }}$ do not exceed the typical value of $\sim 2 \AA$ from McCall (1982).

Since the blue and red spectra have no emission lines in common, the two sets were matched the following way: in the blue wavelength range, the strength of the emission lines was normalized to $\mathrm{H} \beta$, while in the red, the line fluxes are normalized to $\mathrm{H} \alpha$. After reddening correction, the two sides are scaled assuming an intensity ratio of $\mathrm{H} \alpha / \mathrm{H} \beta=2.86$ (Case $\mathrm{B}, T_{\mathrm{e}}=10^{4} \mathrm{~K}$, $n_{\mathrm{e}}=100 \mathrm{~cm}^{-3}$ ). The blue side of our spectra is the one whose flux calibration is taken as reference. 
Table 2. GMOS Spectroscopic setup information.

\begin{tabular}{ccccc}
\hline \hline $\begin{array}{c}\text { Spectral range } \\
(\AA)\end{array}$ & $\begin{array}{c}\text { Dispersion } \\
\left(\AA \mathrm{pix}^{-1}\right)\end{array}$ & Resolving power & $\begin{array}{c}\text { Spatial resolution } \\
\left(\text { ( }^{-1}\right)\end{array}$ & $\begin{array}{c}\text { Exposure time } \\
(\mathrm{s})\end{array}$ \\
\hline $3600-6400$ & 0.90 & 1688 & 0.29 & $3 \times 1200$ \\
$6300-9110$ & 0.94 & 3744 & 0.29 & $3 \times 600$ \\
\hline
\end{tabular}

Table 3. HeII nebulae and dominant ionizing stars.

\begin{tabular}{|c|c|c|c|c|}
\hline \multirow[t]{3}{*}{ HII region } & \multicolumn{4}{|c|}{ Associated ionizing star(s) } \\
\hline & \multirow[t]{2}{*}{ Name $^{a}$} & \multirow[t]{2}{*}{ Other designation ${ }^{b}$} & \multicolumn{2}{|c|}{ Spectral type } \\
\hline & & & Previous work ${ }^{c}$ & This work \\
\hline \multirow{3}{*}{ MA 1} & $\mathrm{~J} 013303.19+301124.2$ & MC8 = M33-WR14 & $\mathrm{WN}$ & WNE \\
\hline & J013303.19+301124.2 & $\mathrm{MC} 9=\mathrm{M} 33-\mathrm{WR} 15$ & WN & WNE \\
\hline & $\mathrm{J} 013302.28+301119.1$ & $\mathrm{~K} 10-01$ & - & WN3-4 \\
\hline \multirow{5}{*}{ BCLMP 49} & J013333.81+304129.6 & NGC 595-WR9 & WN7 & WN7 \\
\hline & J013333.66+304129.8 & $\mathrm{K} 10-02$ & - & $\mathrm{O}$ \\
\hline & J013333.46+304129.8 & & & featureless $^{d}$ \\
\hline & J013333.31+304129.7 & AM5 = M 33-WR45 = NGC 595-WR3 & WC6 & WC6 \\
\hline & J013334.38+304130.2 & $\mathrm{AM} 7=\mathrm{M} 33-\mathrm{WR} 51=$ NGC 595-WR7 & WNL & WN8 \\
\hline BCLMP 40 & J013343.19+303906.2 & "W91 129" (M 33-WR72) & WN7+abs & WN7-8 \\
\hline \multicolumn{5}{|l|}{ HBW 673} \\
\hline \multirow{2}{*}{ BCLMP 720} & J013416.07+303642.1 & H108 & P Cyg LBV candidate ${ }^{e}$ & P Cyg LBV candidate \\
\hline & J013416.28+303646.4 & MC70 = M33-WR129 & WC5 & WC5 \\
\hline \multirow{2}{*}{ BCLMP 715} & J013422.54+303317.1 & MC72 = M33-WR133 & $\overline{\mathrm{WN}}$ & WN4 \\
\hline & $\mathrm{J} 013422.37+303313.7$ & $\mathrm{~K} 10-03$ & - & WN4 \\
\hline BCLMP 651 & $\begin{array}{l}\text { J013429.44+305719.2 } \\
\text { J013429.65+305711.2 }\end{array}$ & & & $\begin{array}{l}\text { featureless }^{d} \\
\text { featureless }^{d}\end{array}$ \\
\hline
\end{tabular}

Notes. ${ }^{(a)}$ LGGS designation (JHHMMSS.ss+DDMMSS.s) from Massey et al. (2006). ${ }^{(b)}$ Star identifications are MC = Massey \& Conti (1983); M 33-WR = Massey \& Johnson (1998; hereafter MJ98); NGC 595-WR = Drissen et al. (1993); AM = Armandroff \& Massey (1985); W91 = see Massey et al. (1995) and MJ98; Hnnn = Corral (1996); K10-nn = this work. ${ }^{(c)}$ Spectral types from MJ98, Abbott et al. (2004), Drissen et al. (2008) and references therein. ${ }^{(d)}$ We are not able to provide a classification for the star since its spectrum shows no obvious stellar features. ${ }^{(e)} \mathrm{P} \mathrm{Cyg} \mathrm{LBV}$ candidate classification comes from Massey et al. (2007).

Reddening-corrected emission-line intensities, normalized to $\mathrm{H} \beta$, are shown in Table 4, together with the values of $\mathrm{C}(\mathrm{H} \beta)$ and $E W_{\text {abs }}$ that provide the best match between the corrected and the theoretical Balmer line ratios. For four objects the uncertainties in $\mathrm{C}(\mathrm{H} \beta)$ are larger than the measured values, and therefore consistent with no reddening. Since [OII] $\lambda 3727$ is the line most affected by extinction in our spectra, for these objects, we checked how much [OII] $\lambda 3727 / \mathrm{H} \beta$ changes with and without the reddening correction. We found that the changes are within the quoted uncertainties.

The line-flux errors are calculated using the expression by Gonzalez-Delgado et al. (1994),

$\sigma_{\text {line }}=\sigma_{\text {cont }} N^{1 / 2}\left(1+\frac{E W}{N \Delta \lambda}\right)^{1 / 2}$

where $\sigma_{\text {cont }}$ is the standard deviation of the continuum near the emission line, $N$ is the width of the region used to measure the line in pixels, $\Delta \lambda$ is the spectral dispersion in $\AA /$ pixel, and $E W$ represents the equivalent width of the line. The final errors quoted in Table 4 account for the uncertainty in the extinction coefficient $\mathrm{C}(\mathrm{H} \beta)$ and include contributions of $<2 \%$ due to the uncertainties in the instrument response fitting. Systematic errors are not included.

\section{Nebular properties}

For our seven HII regions, we calculated the physical properties of the ionized gas in the HeII-emitting zone (see Fig. 3). Physical conditions and chemical abundances for the HII regions are given in Table 5. The quoted uncertainties account for measurement and reddening coefficient errors. Electron densities, electron temperatures and ionic abundances of the ionized gas were derived using the IRAF nebular package (Shaw $\&$ Dufour 1994). We obtained the electron densities, $n_{\mathrm{e}}$, from the $[$ SII] $\lambda 6717 / \lambda 6731$ line ratio. The derived estimates and upper limits for $n_{\mathrm{e}}$ place all of the HII regions in the low-density regime $\left(n_{\mathrm{e}} \lesssim 100 \mathrm{~cm}^{-3}\right)$.

We were able to measure the faint auroral line [OIII] $\lambda 4363$ from the spectra of the three confirmed HeII nebulae: MA 1, BCLMP 651, HBW 673. For these three objects, we derived the electron temperatures $t_{\mathrm{e}}([\mathrm{OIII}])\left(t_{\mathrm{e}}=10^{-4} \times T_{\mathrm{e}}\right)$ using the $[\mathrm{OIII}] \lambda 4363 /[\mathrm{OIII}] \lambda \lambda 4959,5007$ line ratio. For MA 1 , since there is a gradient in $T_{\mathrm{e}}$ between the innermost, HeII-emitting region $(13500 \mathrm{~K})$ and the outer region $(10000 \mathrm{~K})$, we measure the physical properties and chemical abundances from the innermost region. Also, we find MA 1 to be density bounded, and so the ionic species are more co-spatial (see Sect. 4.1.2 for more details on the MA 1 nebula); we therefore derive the chemical abundances of the different elements assuming a single, observed temperature. 
C. Kehrig et al.: Gemini GMOS spectroscopy of HeII nebulae in M33
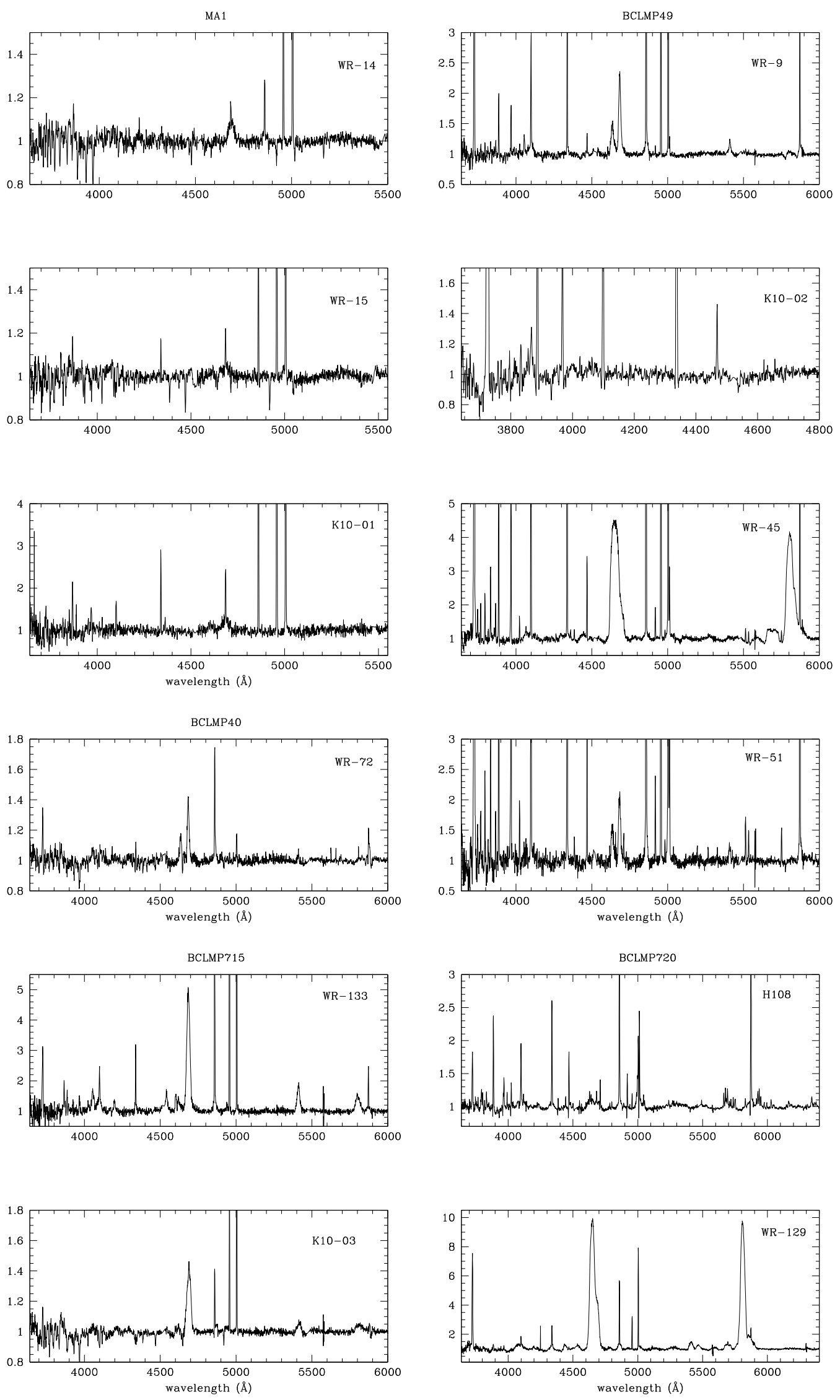

Fig. 4. Rectified blue spectra for the ionizing stars listed in Table 3. 

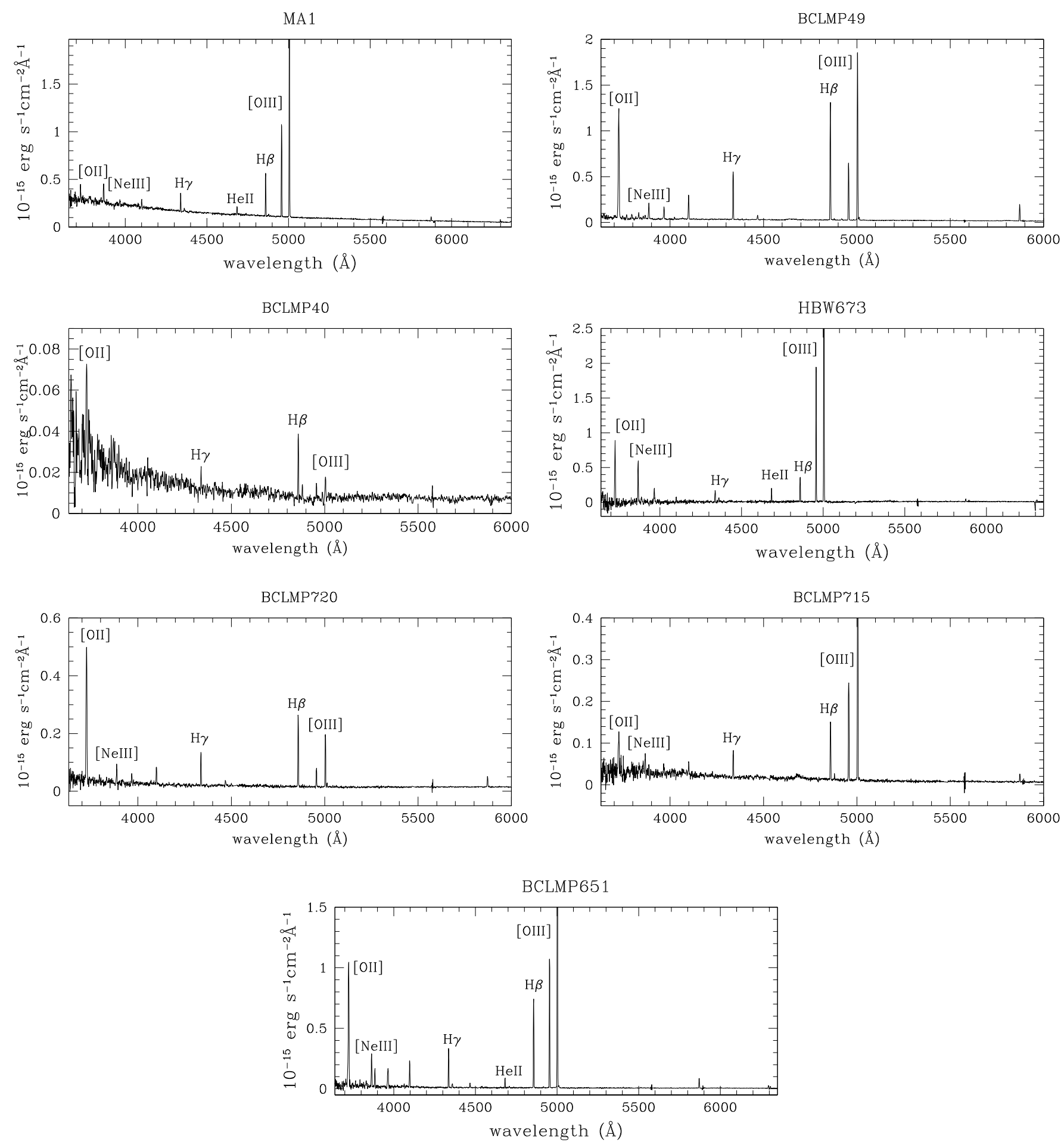

Fig. 5. Blue spectra in units of $10^{-15} \mathrm{erg} \mathrm{s}^{-1} \mathrm{~cm}^{-2} \AA^{-1}$, corresponding to the nebular emission from the zone marked by the yellow box as shown in Fig. 3 for our HII regions. Selected emission lines are labelled.

For BCLMP 651 and HBW673, $t_{\mathrm{e}}([\mathrm{OII}])$ was calculated from the relation between $[\mathrm{OII}]$ and $[\mathrm{OIII}]$ electron temperatures given by Pilyugin et al. (2006): $t_{\mathrm{e}}([\mathrm{OII}])=0.72 t_{\mathrm{e}}([\mathrm{OIII}])+0.26$. Since no auroral line could be measured in the low excitation zone, we assumed the approximation $t_{\mathrm{e}}([\mathrm{SII}]) \approx t_{\mathrm{e}}([\mathrm{NII}])$ $\approx t_{\mathrm{e}}([\mathrm{OII}])$ to be valid. As we did not detect the line [SIII] $\lambda 6312$ in any of our objects, a theoretical relation between [OIII] and [SIII] electron temperatures was used for BCLMP651 and
HBW673: $t_{\mathrm{e}}([\mathrm{SIII}])=1.05 t_{\mathrm{e}}([\mathrm{OIII}])-0.08$ (Pérez-Montero \& Díaz 2005). This relation differs slightly from the empirical relation found by Garnett (1992), mostly due to the introduction of the new atomic coefficients for $S^{2+}$ from Tayal \& Gupta (1999).

In order to derive the chemical abundances we followed as summarized below.

Oxygen. A small fraction of oxygen is expected to be in the form of the $\mathrm{O}^{3+}$ ion in $\mathrm{HII}$ regions which show high-ionization 

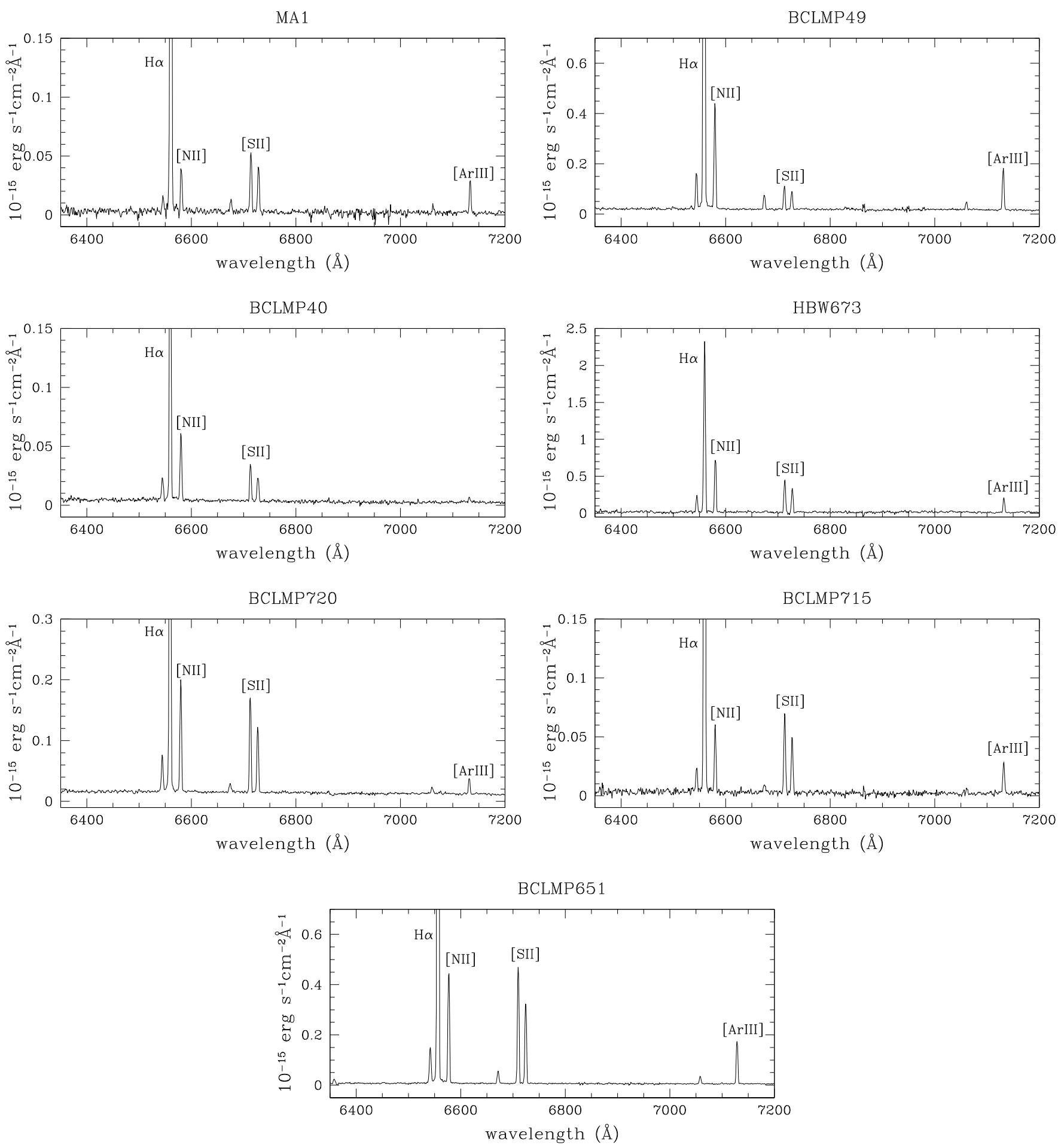

Fig. 6. Red spectra, in units of $10^{-15} \mathrm{erg} \mathrm{s}^{-1} \mathrm{~cm}^{-2} \AA^{-1}$, corresponding to the nebular emission from the zone marked by the yellow box in Fig. 3 for our HII regions. Selected emission lines are labelled.

emission lines like HeII $\lambda 4686$. According to the photoionization models from Izotov et al. (2006b), the $\mathrm{O}^{3+} / \mathrm{O}$ is $\geq 1 \%$ only in the highest-excitation $\mathrm{HII}$ regions for which $\mathrm{O}^{+} /\left(\mathrm{O}^{+}+\mathrm{O}^{2+}\right) \lesssim 10 \%$, as in the case of MA 1 and HBW673. Thus, in order to derive the total oxygen abundances for these two objects, we proceed as following. For MA 1 we assumed $\mathrm{O}^{3+} /\left(\mathrm{O}^{+}+\mathrm{O}^{2+}\right)=4.43 \%$, as obtained from a detailed photoionization model of this object (see Sect. 4.1.2).
In the case of HBW673, we added the fraction of the unseen $\mathrm{O}^{3+}$ ion in its oxygen abundance following the prescription by Izotov et al. (2006b), that gives $\mathrm{O}^{3+} /\left(\mathrm{O}^{+}+\mathrm{O}^{2+}\right) \sim 0.19$. We also checked that using the oxygen ionization correction factor (ICF) from the MA1 photoionization model shifts the total oxygen abundance of HBW673 by 0.06 dex, that is within the quoted uncertainty. For BCLMP651 we set $\mathrm{O}^{3+} / \mathrm{H}^{+}$ to zero, since it shows $\mathrm{O}^{+} /\left(\mathrm{O}^{+}+\mathrm{O}^{2+}\right)>10 \%$, and assume $\mathrm{O} / \mathrm{H}=\left(\mathrm{O}^{+} / \mathrm{H}^{+}\right)+\left(\mathrm{O}^{++} / \mathrm{H}^{+}\right)$. 
A\&A 526, A128 (2011)

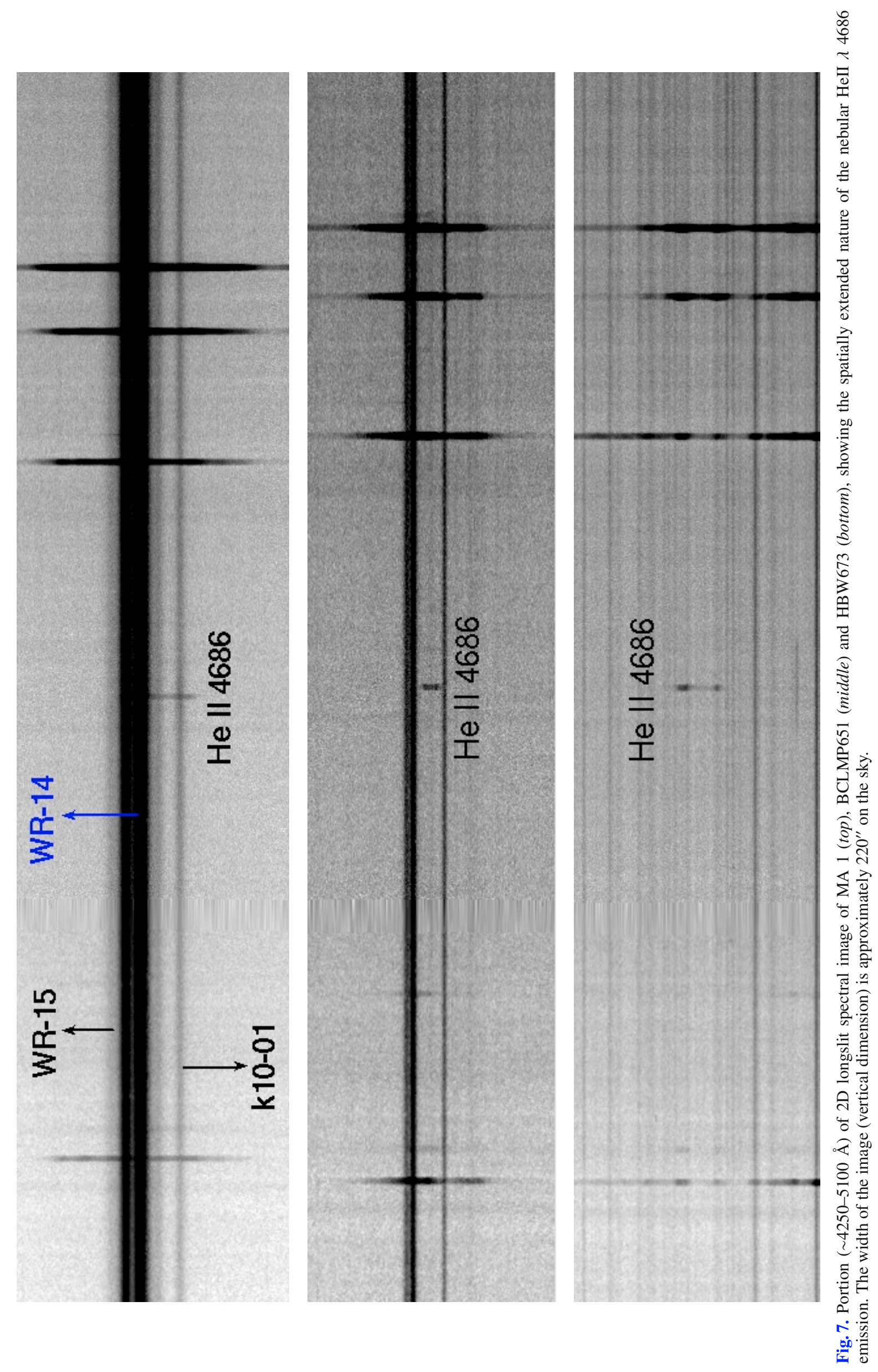


Table 4. Observed HII region line fluxes.

\begin{tabular}{|c|c|c|c|c|c|c|c|}
\hline Wavelength & MA 1 inner $^{a}$ & BCLMP49 & BCLMP40 & HBW673 & BCLMP720 & BCLMP715 & BCLMP651 \\
\hline 3727 [O II] & $54.0 \pm 7.0$ & $172 \pm 7$ & $195 \pm 53$ & $337 \pm 182$ & $280 \pm 28$ & $97.0 \pm 19.0$ & $215 \pm 17$ \\
\hline 3868 [Ne III] & $55.0 \pm 10.0$ & $4.00 \pm 1.00$ & - & $181 \pm 59$ & - & $31.0 \pm 7.0$ & $43.0 \pm 4.0$ \\
\hline $3889 \mathrm{H} 8+\mathrm{He} \mathrm{I}$ & $18.0 \pm 1.0$ & $18.0 \pm 1.0$ & - & - & $23.0 \pm 9.0$ & $14.0 \pm 6.0$ & $21.0 \pm 3.0$ \\
\hline $3968 \mathrm{H} 7+[\mathrm{Ne} \mathrm{III}]$ & $34.0 \pm 6.0$ & $14.0 \pm 1.0$ & - & $77.0 \pm 43.0$ & $18.0 \pm 8.0$ & - & $34.0 \pm 3.0$ \\
\hline $4100 \mathrm{H} \delta$ & $25.0 \pm 4.0$ & $26.0 \pm 2.0$ & - & $24.0 \pm 2.0$ & $26.0 \pm 9.0$ & $26.0 \pm 5.0$ & $29.0 \pm 3.0$ \\
\hline $4340 \mathrm{H} \gamma$ & $49.0 \pm 2.0$ & $47.0 \pm 1.0$ & $47.0 \pm 12.0$ & $48.0 \pm 10.0$ & $47.0 \pm 2.0$ & $47.0 \pm 3.0$ & $46.0 \pm 2.0$ \\
\hline 4363 [O III] & $9.00 \pm 3.00$ & - & - & $18.0 \pm 5.0$ & - & - & $4.70 \pm 0.50$ \\
\hline $4471 \mathrm{He} \mathrm{I}$ & - & $4.00 \pm 0.40$ & - & - & $8.00 \pm 1.00$ & - & $4.60 \pm 0.50$ \\
\hline 4686 He II & $19.0 \pm 2.0$ & - & - & $55.0 \pm 9.0$ & - & - & $11.0 \pm 1.0$ \\
\hline $4861 \mathrm{H} \beta$ & $100 \pm 1$ & $100 \pm 1$ & $100 \pm 6$ & $100 \pm 6.0$ & $100 \pm 2$ & $100 \pm 2$ & $100 \pm 1$ \\
\hline 4959 [O III] & $207 \pm 1$ & $47.0 \pm 1.0$ & $12.0 \pm 5.0$ & $554 \pm 39$ & $25.0 \pm 1.0$ & $160 \pm 5$ & $147 \pm 2$ \\
\hline 5007 [O III] & $602 \pm 30$ & $139 \pm 2$ & $36.0 \pm 6.0$ & $1663 \pm 119$ & $73.0 \pm 2.0$ & $484 \pm 14$ & $440 \pm 7$ \\
\hline $5876 \mathrm{He} \mathrm{I}$ & $9.00 \pm 2.00$ & $11.8 \pm 0.3$ & - & $10.0 \pm 2.0$ & $15.0 \pm 1.0$ & $12.0 \pm 1.0$ & $12.0 \pm 1.0$ \\
\hline 6300 [O I] & - & $0.70 \pm 0.10$ & - & - & $1.70 \pm 0.60$ & $4.00 \pm 1.00$ & $3.20 \pm 0.20$ \\
\hline 6548 [N II] & $3.00 \pm 1.00$ & $8.70 \pm 0.30$ & $20.0 \pm 8.0$ & $26.0 \pm 7.0$ & $17.0 \pm 1.00$ & $8.00 \pm 1.00$ & $8.50 \pm 0.50$ \\
\hline $6563 \mathrm{H} \alpha$ & $286 \pm 6$ & $286 \pm 7$ & $286 \pm 113$ & $286 \pm 79$ & $286 \pm 20$ & $286 \pm 25$ & $286 \pm 16$ \\
\hline 6584 [N II] & $6.00 \pm 1.00$ & $26.0 \pm 1.0$ & $58.0 \pm 23.0$ & $86.0 \pm 24.0$ & $53.0 \pm 4.0$ & $23.0 \pm 3.0$ & $24.0 \pm 1.0$ \\
\hline $6678 \mathrm{He} \mathrm{I}$ & $4.00 \pm 1.00$ & $3.50 \pm 0.20$ & - & - & $4.80 \pm 0.40$ & $3.30 \pm 0.60$ & $2.80 \pm 0.20$ \\
\hline 6717 [S II] & $8.00 \pm 1.00$ & $5.10 \pm 0.30$ & $30.0 \pm 9.0$ & $55.0 \pm 16.0$ & $43.0 \pm 3.0$ & $29.0 \pm 3.0$ & $25.0 \pm 2.0$ \\
\hline 6731 [S II] & $5.00 \pm 1.00$ & $4.00 \pm 0.20$ & $22.0 \pm 8.0$ & $37.0 \pm 11.0$ & $30.0 \pm 2.0$ & $20.0 \pm 2.0$ & $17.0 \pm 2.0$ \\
\hline 7136 [Ar III] & $8.00 \pm 1.00$ & $9.30 \pm 0.40$ & $4.00 \pm 1.00$ & $23.0 \pm 8.0$ & $6.70 \pm 0.70$ & $11.0 \pm 1.0$ & $9.00 \pm 1.00$ \\
\hline 9069 [S III] & $12.0 \pm 4.0$ & $7.40 \pm 0.50$ & $5.00 \pm 2.00$ & $8.00 \pm 4.00$ & $6.00 \pm 1.00$ & $7.00 \pm 3.00$ & $5.00 \pm 1.00$ \\
\hline$C_{\mathrm{H} \beta}$ & $0.09 \pm 0.08$ & $0.37 \pm 0.03$ & $0.22 \pm 0.57$ & $0.09 \pm 0.39$ & $0.12 \pm 0.10$ & $0.05 \pm 0.12$ & $0.04 \pm 0.08$ \\
\hline$E W_{\text {abs }}(\AA)$ & 0.3 & 1.7 & 2.0 & 0.1 & 0.3 & 1.2 & 0.1 \\
\hline
\end{tabular}

Notes. Reddening-corrected line fluxes, normalized to $\mathrm{F}(\mathrm{H} \beta)=100$, for apertures defined by the HeII-emitting region (Fig. 3). The reddening coefficients, $C(\mathrm{H} \beta)$, and the value of the absorption correction, $E W_{\text {abs }}$ are listed in the last two rows for each HII region. The quoted uncertainties include measurement and reddening errors. ${ }^{(a)}$ Emission-line fluxes from the inner, HeII-emitting part of the nebula (see Sect. 4.1.2 for details).

Table 5. Nebular properties.

\begin{tabular}{lccccccc}
\hline \hline Parameter & MA $1^{a}$ & BCLMP 49 & BCLMP 40 & HBW673 & BCLMP 720 & BCLMP 715 & BCLMP651 \\
\hline$n_{\mathrm{e}}([\mathrm{S} \mathrm{II}])\left(\mathrm{cm}^{-3}\right)$ & $<100$ & 159 & $<100$ & $<100$ & $<100$ & $<100$ & $<100$ \\
$t_{\mathrm{e}}([\mathrm{O} \mathrm{III}])\left(10^{4} \mathrm{~K}\right)$ & $1.35 \pm 0.20$ & - & - & $1.19 \pm 0.11$ & - & - & $1.18 \pm 0.04$ \\
$t_{\mathrm{e}}([\mathrm{S} \mathrm{IIII}])\left(10^{4} \mathrm{~K}\right)$ & - & - & - & $1.17 \pm 0.12$ & - & - & $1.17 \pm 0.04$ \\
$t_{\mathrm{e}}([\mathrm{O} \mathrm{II}])\left(10^{4} \mathrm{~K}\right)$ & - & - & - & $1.14 \pm 0.08$ & - & - & $1.12 \pm 0.03$ \\
$12+\log \left(\mathrm{O}^{+} / \mathrm{H}^{+}\right)$ & $6.80 \pm 0.18$ & - & - & $7.82 \pm 0.26$ & - & - & $7.65 \pm 0.05$ \\
$12+\log \left(\mathrm{O}^{2+} / \mathrm{H}^{+}\right)$ & $7.92 \pm 0.15$ & - & - & $8.50 \pm 0.10$ & - & - & $7.94 \pm 0.04$ \\
$12+\log \left(\mathrm{O} / \mathrm{H}^{b}\right.$ & $8.00 \pm 0.05$ & 8.35 & 8.65 & $8.66 \pm 0.09$ & 8.54 & 8.20 & $8.12 \pm 0.03$ \\
$12+\log \left(\mathrm{N}^{+} / \mathrm{H}^{+}\right)$ & $5.77 \pm 0.12$ & - & - & $7.08 \pm 0.11$ & - & - & $6.55 \pm 0.03$ \\
$\log (\mathrm{N} / \mathrm{O})$ & $-1.03 \pm 0.10$ & - & - & $-0.74 \pm 0.28$ & - & - & $-1.10 \pm 0.06$ \\
$12+\log \left(\mathrm{Ne}^{2+} / \mathrm{H}^{+}\right)$ & $7.30 \pm 0.20$ & - & - & $8.01 \pm 0.19$ & - & - & $7.40 \pm 0.06$ \\
$\log (\mathrm{Ne} / \mathrm{O})$ & $-0.62 \pm 0.09$ & - & - & $-0.49 \pm 0.22$ & - & - & $-0.54 \pm 0.07$ \\
$12+\log \left(\mathrm{S}^{+} / \mathrm{H}^{+}\right)$ & $5.19 \pm 0.10$ & - & - & $6 .-19 \pm 0.11$ & - & - & $5.86 \pm 0.03$ \\
$12+\log \left(\mathrm{S}^{2+} / \mathrm{H}^{+}\right)$ & $6.26 \pm 0.09$ & - & - & $6.18 \pm 0.08$ & - & - & $5.98 \pm 0.03$ \\
$12+\log (\mathrm{S} / \mathrm{H})$ & $6.47 \pm 0.10$ & - & - & $6.67 \pm 0.09$ & - & - & $6.26 \pm 0.03$ \\
$12+\log \left(\mathrm{Ar}{ }^{2+} / \mathrm{H}^{+}\right)$ & $5.58 \pm 0.11$ & - & - & $6.17 \pm 0.08$ & - & - & $5.76 \pm 0.03$ \\
$12+\log (\mathrm{Ar} / \mathrm{H})$ & $5.73 \pm 0.11$ & - & - & $6.21 \pm 0.08$ & - & - & $5.79 \pm 0.03$ \\
$\log ([\mathrm{OII}] /[\mathrm{OIII}])^{c}$ & $-1.18 \pm 0.06$ & $-0.03 \pm 0.02$ & $0.61 \pm 0.24$ & $-0.82 \pm 0.24$ & $0.45 \pm 0.04$ & $-0.82 \pm 0.09$ & $-0.44 \pm 0.03$ \\
$\log ([\mathrm{SII}] /[\mathrm{SIII}])^{d}$ & $0.03 \pm 0.07$ & $0.09 \pm 0.03$ & $1.02 \pm 0.14$ & $1.06 \pm 0.11$ & $1.09 \pm 0.04$ & $0.85 \pm 0.21$ & $0.92 \pm 0.03$ \\
\hline
\end{tabular}

Notes. ${ }^{(a)}$ Nebular properties of MA 1 were derived using the emission-line fluxes from the inner, HeII-emitting part of the nebula (see Sect. 4.1 .2 for details). ${ }^{(b)}$ MA 1, HBW673, BCPLM651: O/H abundance derived using $t_{\mathrm{e}}([\mathrm{OIII}])$; BCLMP49, BCLMP40, BCLMP720, BCLMP715: O/H derived from Pettini \& Pagel (2004) - O3N2 method. ${ }^{(c)}$ [OII] $\lambda 3727 /[\mathrm{OIII}] \lambda \lambda 4959,5007 .{ }^{(d)}[\mathrm{SII}] \lambda \lambda 6717,6731 /[\mathrm{SIII}] \lambda 9069$.

To obtain the oxygen abundances in those HII regions in which [OIII] $\lambda 4363$ could not be measured, we applied a commonly used, strong-line method from Pettini \& Pagel (2004): the O3N2 index $\log [([\mathrm{OIII}] \lambda 5007 / \mathrm{H} \beta) /([\mathrm{NII}] \lambda 6584 / \mathrm{H} \alpha)]$, whose associated uncertainty is $\sim 0.25$ dex. Nevertheless one should bear in mind that calibrations of different strong-line indices provide different metallicities (see Bresolin et al. 2009, and references therein for a more detailed discussion).
Sulfur and argon. We derive $\mathrm{S}^{+} / \mathrm{H}^{+}$abundances from the fluxes of the [SII] $\lambda \lambda 6717,6731$ emission lines. Abundances of $\mathrm{S}^{2+} / \mathrm{H}^{+}$were obtained from the flux of the [SIII] $\lambda 9069$ line. We calculate the abundance of $\mathrm{Ar}^{2+} / \mathrm{H}^{+}$from the emission of $[\mathrm{ArIII}] \lambda 7136$, assuming $t_{\mathrm{e}}([\mathrm{ArIII}]) \approx t_{\mathrm{e}}([\mathrm{SIII}])$ (Garnett 1992). To derive the total argon and sulfur abundances for BCLMP651 and HBW673, we adopted the metallicitydependent ICFs of Izotov et al. (2006b). In the case of MA 1, we assume $\mathrm{Ar}^{+} / \mathrm{Ar}^{2+}=0.016, \mathrm{Ar}^{3+} / \mathrm{Ar}^{2+}=0.378$, and 

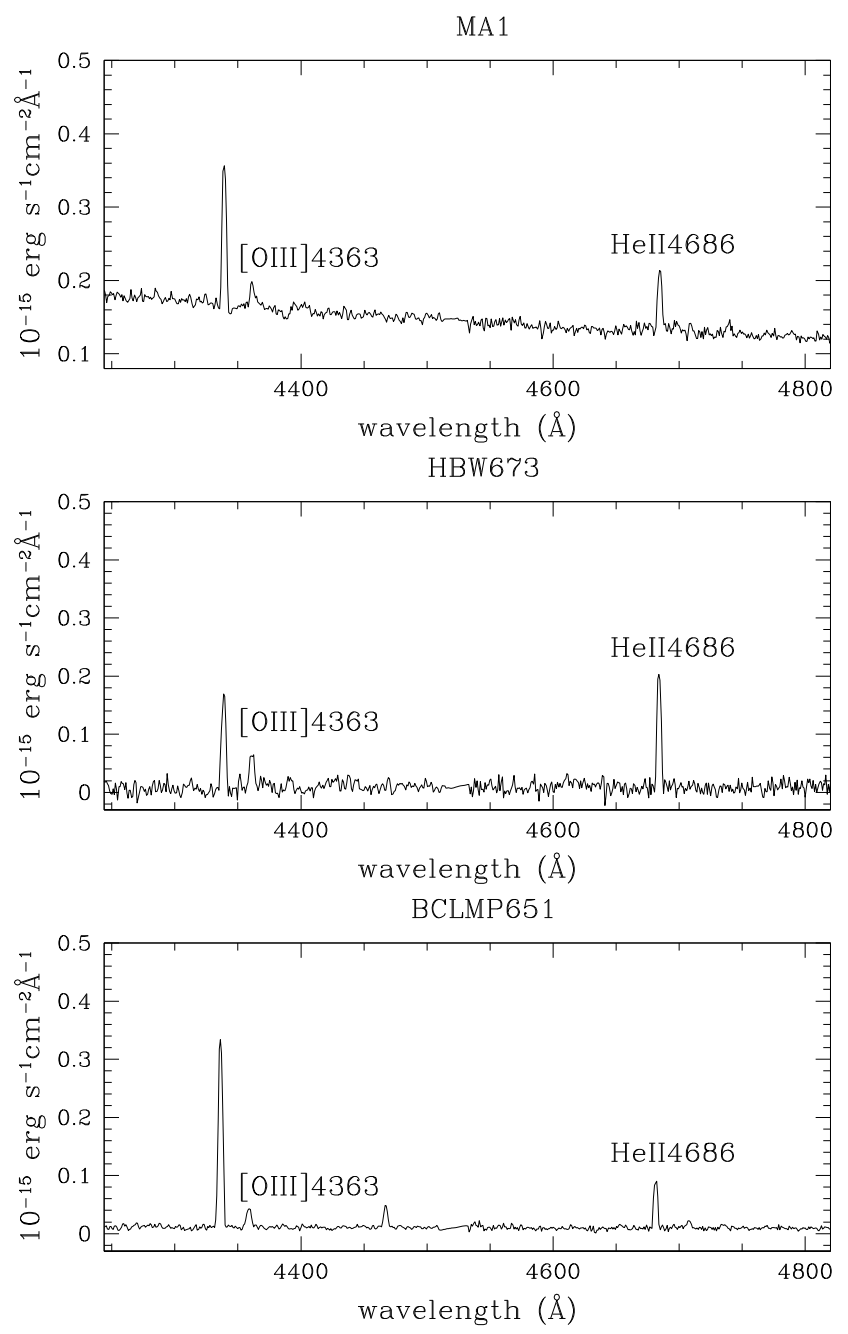

Fig. 8. MA 1, HBW673, BCLMP651: zoom of the wavelength range 4350-4800 ̊ showing the faint, temperature sensitive emission line [OIII] $\lambda 4363$ and the nebular HeII $\lambda 4686$ line.

$\mathrm{S}^{3+} /\left(\mathrm{S}^{+}+\mathrm{S}^{2+}\right)=0.512$, taken from our photoionization modeling of the MA 1 nebula (see Sect. 4.1.2).

Nitrogen and neon. We calculated the $\mathrm{N} / \mathrm{O}$ and $\mathrm{Ne} / \mathrm{O}$ abundance ratios assuming that $\mathrm{N} / \mathrm{O}=\mathrm{N}^{+} / \mathrm{O}^{+}$and $\mathrm{Ne} / \mathrm{O}=\mathrm{Ne}^{++} / \mathrm{O}^{++}$, based on the similarity of the ionization potentials of the ions involved (Peimbert \& Costero 1969). This ICF scheme has been used in previous work (e.g., López-Sánchez et al. 2007; Hägele et al. 2008; Bresolin et al. 2009) and is a good approximation for high ionization degree objects. In our spectra, neon is seen via the [NeIII] emission line at $3869 \AA$. For this ion, we also took the [OIII] electron temperature as representative of the high-excitation zone, in BCLMP 651 and HBW673.

Figure 9 shows the standard [OIII] $\lambda 5007 / \mathrm{H} \beta$ versus [NII] $\lambda 6584 / \mathrm{H} \alpha$ diagnostic diagram (Baldwin et al. 1981; hereafter BPT) for our sample of seven candidates HeII-emitting nebulae. The solid curve, from Kauffmann et al. (2003), separates objects photoionized by purely thermal (stellar) sources from regions that are excited by additional processes. The majority of our objects are found in the zone of objects photoionized by thermal sources. However, HBW 673, one of our HeII nebulae, is the only object located outside of this zone (see Sect. 4.2 for a more detailed discussion of HBW 673).

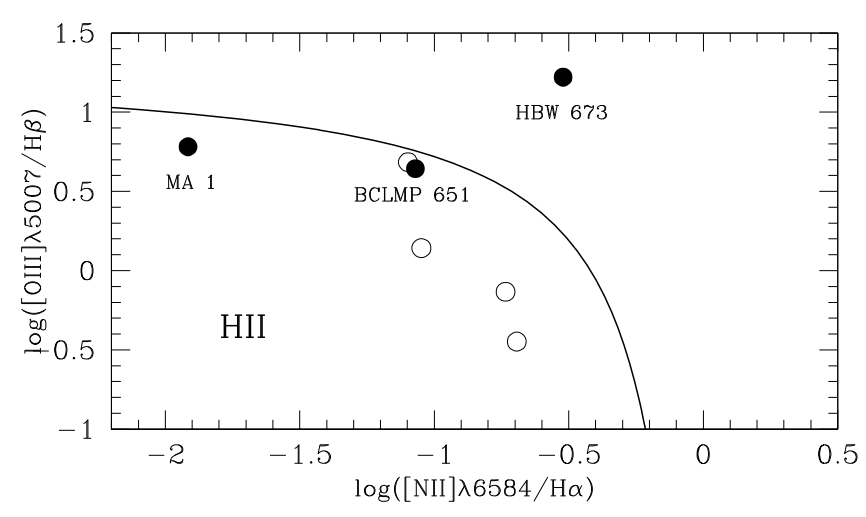

Fig. 9. BPT diagnostic diagram for our sample of seven candidates for HeII-emitting HII regions. Full and empty circles represent the three confirmed HeII nebulae (MA 1, BCLMP 651, HBW 673) and the four remaining objects, respectively. The solid curve, from Kauffmann et al. (2003), indicates the empirical division between objects photoionized by stellar radiation (below the solid curve) and regions that are not dominated by stellar photoionization (above the solid curve).

Table 6. Physical properties of WN atmosphere models for MC8.

\begin{tabular}{lccc}
\hline \hline Parameter & $\log L / L_{\odot}=5.3$ & $\log L / L_{\odot}=5.6$ & $\log L / L_{\odot}=5.9$ \\
\hline$T_{*}(\mathrm{kK})^{a}$ & 90 & 90 & 90 \\
$T_{\text {eff }}(\mathrm{kK})^{a}$ & 78 & 80 & 85 \\
$\mathrm{~d} M / \mathrm{d} t\left(10^{-5} M_{\odot} \mathrm{yr}^{-1}\right)$ & 0.8 & 1.1 & 1.2 \\
$M_{\mathrm{V}}(\mathrm{mag})$ & -3.4 & -4.0 & -4.4 \\
$\log Q_{0}$ & 49.16 & 49.47 & 49.76 \\
$\log Q_{1}$ & 48.92 & 49.24 & 49.55 \\
$\log Q_{2}$ & 38.40 & 46.46 & 48.32 \\
\hline
\end{tabular}

Notes. ${ }^{(a)}$ The stellar temperature, $T_{*}$, is derived from the StefanBoltzmann relation, with the stellar radius defined at a Rosseland optical depth of $\tau=20$. The effective temperature, $T_{\text {eff }}$, is similarly derived, using the effective radius defined at $\tau=2 / 3$.

Table 7. Nebular properties of MA 1 zones.

\begin{tabular}{lcc}
\hline \hline Parameter & Inner region & Outer region \\
\hline $\log n(H)_{0}\left(\mathrm{~cm}^{3}\right)$ & 1.85 & 0.95 \\
$\log r_{0}(\mathrm{~cm})$ & 19.50 & 19.91 \\
$\log r_{\text {outer }}(\mathrm{cm})$ & 19.78 & 20.09 \\
$v_{\text {turb }}\left(\mathrm{km} \mathrm{s}^{-1}\right)$ & 4.0 & 4.0 \\
$12+\log (\mathrm{He} / \mathrm{H})$ & 11.0 & 11.0 \\
$12+\log (\mathrm{O} / \mathrm{H})$ & 8.00 & 8.00 \\
$12+\log (\mathrm{N} / \mathrm{H})$ & 6.97 & 6.97 \\
$12+\log (\mathrm{Ne} / \mathrm{H})$ & 7.38 & 7.38 \\
$12+\log (\mathrm{S} / \mathrm{H})$ & 6.47 & 6.47 \\
$12+\log (\mathrm{Ar} / \mathrm{H})$ & 5.73 & 5.73 \\
\hline
\end{tabular}

\section{Description of the individual Hell nebulae}

\subsection{MA 1}

This object is Number 1 in the list of emission-line nebulae in M 33 by Mayall \& Aller (1942). MA 1 is one of the first HeII nebulae identified in a spiral galaxy beyond the Milky Way (Garnett et al. 1992) and is one of McCall's four "Rosetta Stone" extragalactic HII regions (McCall 1982). It shows a complex morphology of arcs, loops, and filaments having a diameter of $\sim 50^{\prime \prime}$ (200 pc at our assumed distance of $840 \mathrm{kpc}$ ) as seen from the $\mathrm{H} \alpha$ image (see Fig. 3). MA 1 is one of the outermost known 
Table 8. Dereddened line fluxes relative to $F(\mathrm{H} \beta)=100$ for central zone of MA 1 .

\begin{tabular}{lcccc}
\hline \hline Wavelength & MA 1 Inner & Inner model & MA 1 Outer & Outer model \\
\hline $3727[\mathrm{O} \mathrm{II}]$ & $54.0 \pm 7.0$ & 56.0 & $78.6 \pm 1.7$ & 81.7 \\
$3868[\mathrm{Ne} \mathrm{III]}$ & $55.0 \pm 10.0$ & 70.0 & $47.3 \pm 1.0$ & 64.0 \\
$4363[\mathrm{O} \mathrm{III}]$ & $9.00 \pm 3.00$ & 10.00 & $3.50 \pm 0.50$ & 8.00 \\
$4686 \mathrm{He}$ II & $19.0 \pm 2.0$ & 17.0 & $0.10 \pm 0.20$ & 0.30 \\
$4861 \mathrm{H} \beta$ & $100 \pm 1$ & 100 & $100 \pm 1$ & 100 \\
$5007[\mathrm{O} \mathrm{III}]$ & $602 \pm 30$ & 612 & $556 \pm 28$ & 569 \\
$5876 \mathrm{He}$ I & $9.00 \pm 2.00$ & 10.70 & $13.0 \pm 2.6$ & 12.2 \\
$6584[\mathrm{~N} \mathrm{II}]$ & $6.00 \pm 1.00$ & 6.40 & $12.1 \pm 1.2$ & 10.1 \\
$6678 \mathrm{He} \mathrm{I}$ & $4.00 \pm 1.00$ & 3.00 & $2.60 \pm 0.40$ & 3.40 \\
$6717,6731[\mathrm{~S} \mathrm{II}]$ & $13.0 \pm 1.0$ & 13.4 & $26.5 \pm 2.0$ & 19.0 \\
$7136[\mathrm{Ar} \mathrm{III}]$ & $8.00 \pm 1.00$ & 8.40 & $8.90 \pm 0.40$ & 9.30 \\
$9069[\mathrm{~S} \mathrm{III}]$ & $12.0 \pm 4.0$ & 21.1 & $29.1 \pm 1.2$ & 23.6 \\
\hline
\end{tabular}

Table 9. HII regions with nebular He II emission in the Local Group.

\begin{tabular}{|c|c|c|c|c|c|c|}
\hline Galaxy & Region & $12+\log (\mathrm{O} / \mathrm{H})$ & Ionizing source & Spectral type & $\mathrm{HeII} \lambda 4686 / \mathrm{H} \beta[F(\mathrm{H} \beta)=100]$ & References $^{b}$ \\
\hline MW & $\mathrm{G} 2.4+1.4$ & 8.45 & WR102 & WO2 & $40-120$ & $7,3,4,17$ \\
\hline LMC & $\mathrm{N} 44 \mathrm{C}$ & 8.32 & $\mathrm{X}-5 ?$ & X-ray Neb? & $2-14$ & 14,6 \\
\hline LMC & N159 & $8.36^{a}$ & $X-1$ & HMXB & 5 & 13,5 \\
\hline LMC & N79 & $8.17-8.27$ & ВАТ99-2 & WN2b(h) & $21-56$ & 15,11 \\
\hline LMC & N206 & 8.36 & BAT99-49 & WN4:b+O8V & $8-10$ & 12,10 \\
\hline SMC & N76 & 7.93 & $\mathrm{AB} 7$ & WN4+O6I(f) & $16-24$ & $19,5,10$ \\
\hline SMC & NGC 249 & $8.11^{a}$ & SMC-WR10 & WN3ha & & 16 \\
\hline IC1613 & S3 & 7.70 & & WO3 & 23 & $2,5,9$ \\
\hline M33 & BCLMP38b & 8.39 & MC45 & WC4 & 17 & 18,1 \\
\hline M 33 & BCLMP90 & 8.50 & & no obvious hot star associated & 8 & 18 \\
\hline M33 & $\mathrm{C} 001 \mathrm{Ab}$ & 8.61 & & no obvious hot star associated & 2 & 18 \\
\hline M33 & BCLMP208f & 8.07 & & no obvious hot star associated & 3 & 18 \\
\hline M 33 & BCLMP711a & 8.28 & & no obvious hot star associated & 4 & 18 \\
\hline M33 & MA 1 & 8.00 & MC8 & WNE & 19 & 8 \\
\hline M 33 & HBW673 & 8.66 & & no obvious hot star associated & 55 & 8 \\
\hline M 33 & BCLMP651 & 8.12 & & no obvious hot star associated & 11 & 8 \\
\hline
\end{tabular}

Notes. ${ }^{(a)}$ For LMC N159 and SMC NGC 249, since no metallicity measurement has been reported in the literature, we show the average values of the LMC and SMC oxygen abundance from Russell \& Dopita (1990). ${ }^{(b)}$ (1) Abbott et al. (2004); (2) Davidson \& Kinman (1982); (3) Dopita et al. (1990); (4) Esteban et al. (1992); (5) Garnett et al. (1991); (6) Garnett et al. (2000); (7) Johnson (1975); (8) Kehrig et al. (this paper); (9) Kingsburgh \& Barlow (1995); (10) Nazé et al. (2003a); (11) Nazé et al. (2003b); (12) Niemela et al. (1991); (13) Pakull \& Angebault (1986); (14) Pakull \& Motch (1989); (15) Pakull (1991); (16) Pakull (2009); (17) Polcaro et al. (1995); (18) Rosolowsky \& Simon (2008); (19) Testor \& Pakull (1989).

HII regions in M33, located about 30', or $7.3 \mathrm{kpc}$, from the nucleus.

We obtain $12+\log (\mathrm{O} / \mathrm{H}) \sim 8.00$ for MA 1 , consistent with previous work, which demonstrated the low $\mathrm{O} / \mathrm{H}$ of this nebula (e.g., Massey 1985; Garnett et al. 1992; Crockett et al. 2006). The line intensities in this HII region are similar to values seen in metal-poor HII galaxies (see Kehrig et al. 2004, Fig. 14). The presence of HeII $\lambda 4686$ emission in MA 1 was reported by Garnett et al. (1992) and Crockett et al. (2006). The latter showed that the HeII emission is nebular in nature but they did not identify the source of $\mathrm{He}^{+}$-ionizing photons in MA 1 . The high $\log ([\mathrm{OIII}] \lambda 5007 / \mathrm{H} \beta$ ) of $\sim 0.78$ (see Table 4 ), confirms the unsually high excitation and ionization state of this nebula (e.g., RS08), which is unusual for $\mathrm{HII}$ regions with similar $\mathrm{O} / \mathrm{H}$. Our spectrum of MA 1 confirms that the nebular HeII emission is spatially extended (Fig. 7); its observed HeII $\lambda 4686 / \mathrm{H} \beta=0.19$ is consistent with that found for some other metal-poor HII regions excited by WNE stars (e.g., N76 in the SMC; see Table 9).

\subsubsection{Stellar atmosphere models for the WN source MC8}

Here, we investigate whether the early-type WN star MC8 (Massey \& Conti (1983) alias M33-WR14 (MJ98)), is capable of supplying the high energy photons required for He II nebular emission from MA 1. Stellar atmosphere models are constructed using the code CMFGEN (Hillier \& Miller 1998), which solves the radiative transfer equation in the co-moving frame, under the additional constraints of statistical and radiative equilibrium.

From Fig. 3, the early type WN source MC8 is by far the brightest source within the MA 1 nebula. Indeed, ground-based photometry from Massey et al. (2006) indicates $V=16.14 \mathrm{mag}$, from which $E(B-V)=0.06 \mathrm{mag}\left(\right.$ via $\left.C_{\mathrm{H} \beta}=0.09\right)$ implies $M_{\mathrm{V}}=$ -8.7 mag for our assumed distance of $840 \mathrm{kpc}$. In contrast, absolute magnitudes of WN-type stars in the Large Magellanic Cloud range from -2 to -7.5 mag (Crockett et al. 2007), with $M_{\mathrm{V}}=-4$ mag typical for single early-type WN stars. In addition, the stellar He II $\lambda 4686$ equivalent width is $W_{\lambda} \sim 3 \AA$, versus 30-500 $\AA$ for LMC single, early-type WN stars (Crowther $\&$ Hadfield 2006). The properties of MC8 are therefore consistent with a WN star located within a compact, young star cluster such as Brey 65 in the LMC (Walborn et al. 1999), in which the He II $\lambda 4686$ emission strength is severely diluted by other cluster members (mostly O, B and A stars). Alternatively, could MC8 be an Of supergiant, since they possess much weaker He II $\lambda 4686$ emission than WN stars plus brighter absolute 


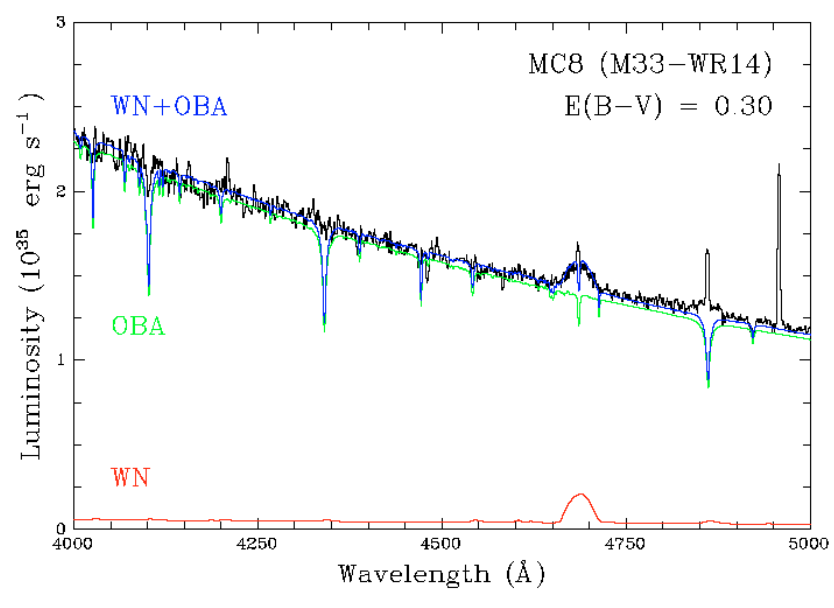

Fig. 10. Comparison between the GMOS spectroscopy of MC8 (M 33WR14), dereddened by $E(B-V)=0.30$ mag and synthetic spectra for the WN component (red, $\log L / L_{\odot}=5.9$ ), OBA cluster from starburst99 (green) and composite thereof (blue).

magnitudes of $M_{\mathrm{V}}=-7 \mathrm{mag}$. We are able to discriminate between these two possibilies since Of supergiants possess narrow $\lambda 4686$ emission, with $F W H M \sim 2-7 \AA$ (Crowther et al. 2002b) versus $F W H M \sim=25-60 \AA$ for early-type WN stars (Crowther $\&$ Hadfield 2006). From our MC8 Gemini GMOS spectrum, He II $\lambda 4686$ emission is broad ( $F W H M \sim 30 \AA$ ), allowing us to conclude that MC8 is a probable cluster hosting an early-type WN star.

Therefore, in our analysis of MC8 we are unable to fix the absolute magnitude of the early-type WN star, and instead focus upon reproducing the line luminosity of He II $\lambda 4686$. We have convolved the GMOS spectrum of MC8 with standard filters, from which synthetic photometry of $V=17.35 \mathrm{mag}$ (and $B-V=0.09 \mathrm{mag}$ ) is obtained, indicating that $\sim 50 \%$ of the continuum light from the MC8 source is included within the slit (recall Fig. 3). Fortunately, since the WN star is included, we are able to estimate its He II $\lambda 4686$ line luminosity. To assess the extinction, we have compared the GMOS spectrum to a 3 Myr old, instantaneous burst, LMC metallicity starburst 99 (Leitherer et al. 1999) evolutionary synthesis model. An extinction of $E(B-V)=0.30$ mag is required to reproduce the spectral energy distribution of the GMOS dataset (Fig. 10), from which we obtain a $\lambda 4686$ line luminosity of $4.5 \times 10^{35} \mathrm{erg} \mathrm{s}^{-1}$ for the WN star, versus an average of $8.4 \pm 7.1 \times 10^{35} \mathrm{erg} \mathrm{s}^{-1}$ for LMC early-type WN stars (Crowther \& Hadfield 2006).

In addition, we are unable to derive a stellar temperature for the WN star since $\lambda 4686$ is the sole diagnostic available to us. In view of the limitations imposed by the sparse continuum and line constraints, we have calculated generic stellar atmosphere models for the WN star hosted by MC8 using CMFGEN. Our approach mimics the study of HD 9974 (WN3) by Marchenko et al. (2004) except for more extensive model atoms: H I, He I-II, C IV, N III-V, O III-VI, Ne IV-V, Si IV, S IV-V, Ar V-VII, Ca V-VII, Fe IV-VII, Ni IV-VII, totalling 822 super-levels (23,098 lines). Other than $\mathrm{H}, \mathrm{He}, \mathrm{CNO}$ we adopt solar abundances (Asplund et al. 2009), scaled to $0.3 Z_{\odot}$ (see Table 5). In the absence of CNO features, we adopt equilibrium WN abundances, namely $X(N)=0.2 \%, \mathrm{X}(\mathrm{C})=\mathrm{X}(\mathrm{O})=0.03 \%$. With regard to wind clumping (Hillier 1991), this is incorporated using a radiallydependent volume filling factor, $f$, with $f_{\infty}=0.1$ at terminal velocity $v_{\infty}$, resulting in a reduction in mass-loss rate by a factor of $\sqrt{(1 / f)} \sim 3$.
In the absence of Wolf-Rayet temperature diagnostics from our GMOS dataset of MC8, Rühling (2008) has undertaken spectral analyses of all WN stars in the LMC including $50 \mathrm{WN} 3-$ 4 stars. From this comprehensive study, an average of $T_{*}=$ $90 \pm 30 \mathrm{kK}$ was obtained that we shall adopt here for the WN star in MC8. As discussed previously (e.g., Schmutz et al. 1992; Schaerer 2002), the wind density of WN atmospheres can dictate the hardness of their ionizing fluxes more significantly than stellar temperature. We demonstrate this effect by selecting three stellar luminosities of the WN component, namely $\log L / L_{\odot}=$ 5.3, 5.6 and 5.9, which correspond to stellar masses of 10,16 and $25 M_{\odot}$ (Schaerer \& Maeder 1992), spanning a representative range for WN stars (Crowther 2007). For these three cases, the (clumped) mass-loss rate required to reproduce the He II $\lambda 4686$ feature was relatively uniform, $0.8,1.1,1.2 \times 10^{-5} M_{\odot} \mathrm{yr}^{-1}$, respectively, with $v_{\infty} \sim 1750 \mathrm{~km} \mathrm{~s}^{-1}$ for each case. However, since the stellar luminosity varies by a factor of four, the wind becomes progressively more transparent at higher luminosity, hardening the ionizing spectrum, as revealed by the number of $\mathrm{He}^{+}$ionizing photons, $Q_{2}$, shown in Table 6 for each model atmosphere. In Fig. 10 we compare the de-reddened GMOS spectrum of MC8 with both the cluster spectral energy distribution and high luminosity WN model.

\subsubsection{Photoionization modeling of the MA 1 nebula}

We now model the MA1 HII region with the photoionization code CLOUDY, v. C08.00 (Ferland et al. 1998), using these stellar atmosphere models for MC8 to define the ionizing spectral energy distribution. While we do not know which, if any, of the three atmosphere models is representative of MC8, our purpose here is to investigate feasibility. We adopt the measured chemical abundances in the central region listed in Table 5, which are consistent with the reported abundance gradient in M33 (e.g., RS08). He II emission is emitted fairly uniformly within the inner nebula, $22 \mathrm{pc}$ in radius. In addition, the outer region of the nebula has a complex morphology extending up to a radius of 60 pc (see Figs. 2 and 7 (top panel)). To account for these distinct nebular components we construct a two-zone photoionization model, composed of an inner and outer region, each with a constant density listed in Table 7. A constant cosmic ray energy density of $2.6 \times 10^{-9} \mathrm{~cm}^{-3}$ (Williams et al. 1998) is included, but is negligible.

Of the three atmosphere models, solely the model illuminated by the highest luminosity $\left(\log L / L_{\odot}=5.9\right)$ atmosphere is capable of reproducing the observed HeII emission, for the observed ionization parameters $\log U \geq-2.0$, where $U=$ $Q^{0} / 4 \pi r^{2} c n_{\mathrm{e}}, Q^{0}$ is the H-ionizing photon emission rate, and $r$ is the Strömgren radius. The parameters for the two parts of this model nebula are listed in Table 7 .We self consistently use the emergent radiation transmitted through the highly ionized gas of the inner region as the ionization source of the outer region beyond the He II emission, accounting for an observed difference in $T_{\mathrm{e}}$ between the inner region $(13500 \mathrm{~K})$ and outer region $(10000 \mathrm{~K})$. The two lower-luminosity $\mathrm{WN}$ atmospheres predict the nebular intensity of $\mathrm{HeII} \lambda 4686 / \mathrm{H} \beta$ to be orders of magnitude lower than observed, and they are not capable of producing nebular He II $\lambda 4686$ emission with realistic choices of metallicity and $U$.

Table 8 shows good agreement between the observed nebular line strengths and the photoionization model. The standard deviation between the observed and predicted emission is $17 \%$, excluding [SIII] $\lambda 9069$, which is affected by telluric absorption. These models demonstrate that the SED of the 
$\log L / L_{\odot}=5.9$ atmosphere is a plausible ionization source for the surrounding nebula, without a need to invoke additional ionization sources. We note that the central line of sight is optically thin: the 2-D morphology revealed by the images and the weakness of $[\mathrm{OII}]$ relative to [OIII] indicates that an $\mathrm{H}^{+}$ionization front is not present in the line of sight toward the He II nebula, consistent with our model. Early spectral observations by McCall (1982) also suggested parts of the nebula to be density bounded based on the [OII] / [OIII] ratio.

While neither our model stellar atmosphere nor model nebula is necessarily meant be an accurate physical representation of this region, the reasonable agreement demonstrates that the observed star is indeed capable of reproducing the observed nebula.

\subsection{HBW673}

HBW673 is a relatively faint and small HII region about $2 \mathrm{kpc}$ from the center of the galaxy. Its diameter is roughly $20^{\prime \prime}(\sim 80 \mathrm{pc}$ at our assumed distance of $840 \mathrm{kpc}$ ), as measured from the $\mathrm{H} \alpha$ image, and in contrast to MA 1, HBW673 does not show a complex morphology (see Fig. 3). From Fig. 7 (bottom) we can see that there is no obvious star associated with HBW673. The spectrum of HBW673 has very strong emission at $\lambda 4686$ showing one of the highest values of HeII $\lambda 4686 / \mathrm{H} \beta=0.55 \mathrm{ob}-$ served among the local HeII nebulae (see Table 9). In addition, HBW673 exhibits extremely high excitation in [OIII] $\lambda 5007$ and in [NeIII] $\lambda 3868$ (see Table 4).

The spectrum of HBW673 is not that of a SNR, lacking the variety of different ions that are shock-excited in classical SNRs (e.g., Blair et al. 1981). A PN-type nature of HBW673 is also ruled out. The typical size of a Galactic PN is some tenths of a parsec (e.g., Peimbert 1990), while evolved PNe have been found with sizes of up to 4 pc (e.g., Corradi et al. 1997). At our assumed distance of $840 \mathrm{kpc}$ to M 33, 1" corresponds to $4 \mathrm{pc}$ and therefore any extended emission region in our images is not considered to be a PN. Such high relative fluxes of the HeII $\lambda 4686$ emission line indicate that radiation with photon energies greater than 4 Rydberg is intense, and difficult to explain by ionizing stellar radiation (e.g., Thuan \& Izotov 2005; Izotov et al. 2004). In addition, the BPT diagnostic diagram (Fig. 9) for our sample of HII regions shows that HBW 673 is outside the zone of normal HII regions. This suggests that the main excitation mechanism in HBW673 is not a hot stellar continuum, but another ionization source. HBW673 is not coincident with any known X-ray sources.

\subsection{BCLMP651}

The third HeII nebula we identified is BCLMP651 with a diameter of $\sim 30^{\prime \prime}$ (120 pc at our assumed distance of $840 \mathrm{kpc}$ ). The nebular morphology consists of a bright knot on the southeast side, and a second, fainter one on the northwest side of the nebula. For BCLMP651 there does not appear to be any WR star responsible for the extended HeII emission. Figure 7 (middle) shows that the nebular HeII emission is placed between two continuum sources. We were not able to detect any obvious stellar features in our spectra of these objects, which only have $S / N \sim 10$. Like the other HeII nebulae, BCLMP651 is also a high-excitation object, with e.g., high [NeIII] $\lambda 3868 / \mathrm{H} \beta=0.40$, in comparison to the other four HII regions for which we do not see nebular HeII emission (see Table 4). This object also is not coincident with any known X-ray source.

\section{Discussion}

From our sample of seven candidate HeII nebulae in M33, we confirm the presence of nebular HeII $\lambda 4686$ emission line in the spectra of three objects: MA 1, HBW673 and BCLMP651. In addition to MA 1 , there are four more confirmed He II nebulae ionized by WN stars in the Local Group: LMC N79 (Pakull 1991), LMC N206 (Niemela et al. 1991), SMC N76 (Testor \& Pakull 1989; Garnett et al. 1991) and NGC 249 (Pakull 2009) in the SMC. However, WN stars are not the only sources capable to ionize HeII. The Galactic nebula G2.4+1.4 and the S3 nebula in IC 1613 exhibit nebular HeII emission associated with WO stars (Johnson 1975; Dopita et al. 1990; Davidson \& Kinman 1982; Garnett et al. 1991), while the nebular He II emission in LMC N44C is puzzling since emission is centered on an $\mathrm{O} 7$ star (Garnett et al. 2000) which is not expected to be hot enough to produce $\mathrm{He}^{+}$-ionizing photons. Pakull \& Motch (1989) have proposed N44C to be a fossil X-ray ionized nebula, but a clearer view of this object is still needed (see Garnett et al. 2000).

Among our sample, the HeII nebulae HBW673 and BCLMP651 are also intriguing objects. The former does not seem to be associated with any hot star. Yet, except for the presence of strong nebular HeII emission, it shows a fairly normal, highly excited, HII region spectrum. Moreover, electron temperatures in these nebulae are not unusally high $(\sim 12000 \mathrm{~K})$.

RS08 have analysed Keck spectra of 61 HII regions in M33, and detected HeII $\lambda 4686$ emission from five of these objects (BCLMP38b, C001Ab, BCLMP711a, BCLMP90, BCLMP208f). They claim the HeII $\lambda 4686$ emission is almost certainly nebular as it is spatially resolved in these five HII regions. Their strongest source, BCLMP38b, was also detected in our continuum-subtracted He II image, but it was compact and unresolved, and so we were not able to identify it as an extended He II nebula. Magrini et al. (2007) also report the existence of HeII emission in three M 33 HII regions (BCLMP258b, BCLMP220, BCLMP45). Doing a coordinate cross-reference with the catalog of WR stars in M33 (Massey et al. 2006), we find that with the exception of BCLMP38b, no HeII nebula presented by RS08 and Magrini et al. (2007) appears to be associated with a WR star. The object BCLMP38b appears to be associated with the WC star MC45 (M33-WR76), which might be responsible for the ionization in the nebula, although no WC stars are currently known to ionize He II nebulae. A more detailed study is necessary to assess whether MC45 is the He IIionizing source.

Table 9 summarizes the HII regions with confirmed nebular He II $\lambda 4686$ emission detected so far in the Local Group. The ionizing sources are apparently quite heterogenous. As discussed previously, the HeII-ionizing sources of M33 HBW673, M 33 BCLMP651 and LMC N44C are still unknown. In several studies of nebular HeII emission in BCDs, Izotov and collaborators (e.g., Thuan \& Izotov 2005; Izotov et al. 2006a) find the presence of nebular HeII $\lambda 4686$ emission even at large distances from young clusters, indicating that mechanisms other than stellar photoionization (e.g., shocks or high-mass X-ray binaries) seem to be present, at least in some objects. A similar result was found by Monreal-Ibero et al. (2010). These authors studied the ionized gas and massive stellar population in the HII galaxy NGC 5253, and found that the nebular HeII $\lambda 4686$ emissions to be, in general, not coincident with WR stars. One can easily envision a mixture of different contributions, including possibly from SNRs, to the observed HeII emission beyond the Local Group. We should also stress that from our survey for HeII nebulae in 
M33, we identified five SNRs with HeII emission. Thus, SNRs or shock-excited objects (e.g., Pakull et al. 2010) can also be responsible for some HeII nebulae.

Even among the WR HeII nebulae, the ionizing stars show different spectral types (Table 9) and at present, it is not possible to look for systematic properties that differentiate the HeIIionizing WR stars from the remainder of them due to the relatively small number of local WR HeII nebulae detected so far. However, an association of nebular HeII emission with WR stars (e.g., MA 1) in low-metallicity environments seems to exist. Model atmospheres of Smith et al. (2002) predict that low wind densities favour transparent winds such that only weak winds are expected to produce a significant $\mathrm{He}+$ continuum below $228 \AA$. Since mass-loss rates of WR stars are predicted (Vink \& de Koter 2005) and observed to scale with metallicity (Crowther et al. 2002a), it is expected that HeII nebulae are preferentially associated with WR stars in metal-poor regions. Indeed, seven of the eight WR stars which exhibit nebular HeII are in nebulae with $12+\log (\mathrm{O} / \mathrm{H})<8.4$ (see Table 9$)$.

\section{Summary}

To investigate the properties of HeII nebulae and the origin of the nebular HeII $\lambda 4686$ emission, we have carried out a survey for HeII nebulae in the Local Group galaxy M33. Due to its proximity, M 33 allows HII regions excited by few/single stars to be resolved. Since M 33 shows a well defined metallicity gradient, this galaxy also offers the opportunity to probe the metallicity dependence of the nebular HeII line formation and strength. The rarity of HII regions which show nebular HeII among Local Group galaxies illustrates the significance of such regions within M33.

From our HeII narrow-band imaging of M33, we identified seven candidates for HeII nebulae, for which we obtained follow-up optical spectra. We detect nebular HeII emission in the spectra of three of the seven candidate HII regions: MA 1, BCLMP651, and HBW673, the latter two being newly confirmed HeII nebulae. All three are high-excitation HII regions.

We have assessed whether the observed early-type WN star MC8 is a plausible source for the hard ionizing radiation of MA 1 through a combined stellar and nebular analysis. A high luminosity, relatively transparent $\mathrm{WN}$ wind is capable of reproducing the nebular properties, although MC8 is almost certainly an unresolved star cluster (cf. Brey 65 and Brey 73 in LMC; Walborn et al. 1999).

The absence of a hot WR star in BCLMP651 and HBW673 suggests possible similarities with N44C and N159 in the LMC, where the high excitation is linked to possible X-ray sources within the nebulae. To date, however, no X-ray counterpart has been linked to either HBW673 or BCLMP651. In HBW673, the highest-excitation object in our sample, the presence of a hard radiation source other than a hot stellar continuum is indicated by the BPT diagnostic diagram (Fig. 9). At present we are unable to identify the likely source powering the emission in BCLMP 651 and HBW 673. Further study of these two objects, which contribute to the heterogeneity of ionizing sources, is required. Additional study of local HII regions with He II emission are needed to lead us to a clearer understanding of the sources of nebular HeII emission before their applicability to starbursts can be properly assessed.

Acknowledgements. Our gratitude to the NOAO Gemini Science Center and Gemini Observatory staff, including Kevin Volk, Dara Norman and Jennifer
Holt. We wish to thank the anonymous referee for his/her useful comments and suggestions. M.S.O. and C.K. acknowledge support from the National Science Foundation, grants AST-0448893 and AST-0806476. C.K., as a Humboldt Fellow, acknowledges support from the Alexander von Humboldt Foundation, Germany. O.S. acknowledges support from the Science and Technology Facilities Council, UK. D.S. is supported by the Swiss National Science Foundation. We thank E. Rosolowsky and J. D. Simon for providing us with their Keck/LRIS datasets. C.K. also thanks Manfred W. Pakull, Hans Zinnecker, José M. Vílchez and Enrique Pérez-Montero for the careful reading of the manuscript and helpful comments. This work is based on observations obtained in programs GN-2006B-Q83 and GN-2008B-C5, at the Gemini Observatory, which is operated by AURA under a cooperative agreement with the NSF on behalf of the Gemini partnership: the National Science Foundation (United States), the Science and Technology Facilities Council (United Kingdom), the National Research Council (Canada), CONICYT (Chile), the Australian Research Council (Australia), CNPq (Brazil) and CONICET (Argentina).

\section{References}

Abbott, J. B., Crowther P. A., Drissen, L., et al. 2004, MNRAS, 350, 552 Armandroff, T. E., \& Massey, P. 1985, ApJ, 291, 685

Asplund, M., Grevesse, N., Sauval, A. J., \& Scott, P. 2009, ARA\&A, 47, 481 Baldwin, J. A., Phillips, M. M., \& Terlevich, R. 1981, PASP, 93, 5

Blair, W. P., Kirshner, R. P., \& Chevalier, R. A. 1981, ApJ, 247, 879

Boulesteix, J., Courtes, G., Laval, A., Monnet, G., \& Petit, H. 1974, A\&A, 37, 33

Bresolin, F., Gieren, W., Kudritzki, R.-P., Pietrzyński, G., et al. 2009, ApJ, 700, 309

Brinchmann, J., Kunth, D., \& Durret, F. 2008, A\&A, 485, 657

Cardelli, J. A., Clayton, G. C., \& Mathis, J. S. 1989, ApJ, 345, 245

Cerviño, M., Mas-Hesse, J. M., \& Kunth, D. 2002, A\&A, 392, 19

Corradi, R. L. M., Perinotto, M., Schwarz, H. E., \& Claeskens, J.-F. 1997, A\&A, 322,975

Corral, L. J. 1996, AJ, 112, 1450

Crockett, N. R., Garnett, D. R., Massey, P., \& Jacoby, G. 2006, ApJ, 637, 741

Crockett, R. M., Smartt, S. J., Eldridge, J. J., Mattila, S., et al. 2007, MNRAS, 381,835

Crowther, P. A. 2007, ARA\&A, 45, 177

Crowther, P. A., \& Hadfield, L. J. 2006, A\&A, 449, 711

Crowther, P. A., Hillier, D. J., \& Smith, L. J. 1995, A\&A, 293, 403

Crowther, P. A., De Marco, O., \& Barlow, M. J. 1998, MNRAS, 296, 367

Crowther, P. A., Pasquali, A., De Marco, O., et al. 1999, A\&A, 350, 1007

Crowther, P. A., Dessart, L., Hillier, D. J., Abbott, J. B., \& Fullerton, A. W. 2002a, A\&A, 392, 653

Crowther, P. A., Hillier, D. J., Evans, C. J., et al. 2002b, ApJ, 579, 774

Davidson, K., \& Kinman, T. D. 1982, PASP, 94, 634

Dawson, S., Rhoads, J. E., Malhotra, S., Stern, D., et al. 2004, ApJ, 617, 707

de Mello, D. F., Schaerer, D., Heldmann, J., \& Leitherer, C. 1998, ApJ, 507, 199

Dopita, M. A., McGregor, P. J., Rawlings, S. J., \& Lozinskaia, T. A. 1990, ApJ,

351,563

Drissen, L., Moffat, A. F. J., \& Shara, M. M. 1993, AJ, 105, 1400

Drissen, L., Crowther, P. A., Ubeda, L., \& Martin, P. 2008, MNRAS, 389, 1033

Esteban, C., Vilchez, J. M., Smith, L. J., \& Clegg, R. E. S. 1992, A\&A, 259, 629

Ferland, G. J., Korista, K. T., Verner, D. A., et al. 1998, PASP, 110, 761

Freedman, W. L.,Madore, B. F., Gibson, B. K., et al. 2001, ApJ, 553, 47

Garnett, D. R. 1992, AJ, 103, 1330

Garnett, D. R., Kennicutt, R. C., Jr., Chu, Y.-H., \& Skillman, E. D. 1991, ApJ, 373,458

Garnett, D. R., Odewahn, S. C., \& Skillman, E. D. 1992, AJ, 104, 1714

Garnett, D. R., Galarza, V. C., \& Chu, Y.-H. 2000, ApJ, 545, 251

Gonzalez-Delgado, R. M., Perez, E., Tenorio-Tagle, G., et al. 1994, ApJ, 437, 239

Guseva, N. G., Izotov, Y. I., \& Thuan, T. X. 2000, ApJ, 531, 776

Hägele, G. F., Díaz, Á. I., Terlevich, E., et al. 2008, MNRAS, 383, 209

Hamann, W.-R., \& Koesterke, L. 1998, A\&A, 333, 251

Hillier, D. J. 1991, A\&A, 247, 455

Hillier, D. J., \& Miller, D. L. 1998, ApJ, 496, 407

Hodge, P. W., Balsley, J., Wyder, T. K., \& Skelton, B. P. 1999, PASP, 111, 685

Hook, I. M., Jørgensen, I., Allington-Smith, J. R., et al. 2004, PASP, 116, 425

Izotov, Y. I., Noeske, K. G., Guseva, N. G., et al. 2004, A\&A, 415, L27

Izotov, Y. I., Schaerer, D., Blecha, A., et al. 2006a, A\&A, 459, 71

Izotov, Y. I., Stasińska, G., Meynet, G., Guseva, N. G., \& Thuan, T. X. 2006b, A\&A, 448, 955

Johnson, H. M. 1975, ApJ, 198, 111

Kauffmann, G., Heckman, T. M., Tremonti, C., et al. 2003, MNRAS, 346, 1055

Kehrig, C., Telles, E., \& Cuisinier, F. 2004, AJ, 128, 1141

Kehrig, C., Vílchez, J. M., Sánchez, S. F., et al. 2008, A\&A, 477, 813

Kingsburgh, R. L., \& Barlow, M. J. 1995, A\&A, 295, 171 
Kobulnicky, H. A., \& Skillman, E. D. 1996, ApJ, 471, 211

Kobulnicky, H. A., Kennicutt, R. C., Jr., \& Pizagno, J. L. 1999, ApJ, 514, 544

Kudritzki, R. P. 2002, ApJ, 577, 389

Kunth, D., \& Sargent, W. L. W. 1981, A\&A, 101, L5

Leitherer, C., Schaerer, D., Goldader, J. D., et al. 1999, ApJS, 123, 3

López-Sánchez, Á. R., \& Esteban, C. 2010, A\&A, 516, A104

López-Sánchez, Á. R., Esteban, C., García-Rojas, J., Peimbert, M., \& Rodríguez, M. 2007, ApJ, 656, 168

Magrini, L., Vílchez, J. M., Mampaso, A., Corradi, R. L. M., \& Leisy, P. 2007, A\&A, 470, 865

Marchenko, S. V., Moffat, A. F. J., Crowther, P. A., et al. 2004, MNRAS, 353, 153

Massey, P. 1985, PASP, 97, 5

Massey, P., \& Conti, P. S. 1983, ApJ, 273, 576

Massey, P., \& Johnson, O. 1998, ApJ, 505, 793

Massey, P., Armandroff, T. E., Pyke, R., Patel, K., \& Wilson, C. D. 1995, AJ, 110,2715

Massey, P., Olsen, K. A. G., Hodge, P. W., et al. 2006, AJ, 131, 2478

Massey, P., McNeill, R. T., Olsen, K. A. G., et al. 2007, AJ, 134, 2474

Mayall, N. U., \& Aller, L. H. 1942, ApJ, 95, 5

McCall, M. L. 1982, Ph.D. Thesis, University of Texas

Monreal-Ibero, A., Vilchez, J. M., Walsh, J. R., \& Munoz-Tunon, C. 2010, A\&A, 517, A27

Nagao, T., Sasaki, S. S., Maiolino, R., et al. 2008, ApJ, 680, 100

Nazé, Y., Rauw, G., Manfroid, J., Chu, Y.-H., \& Vreux, J.-M. 2003a, A\&A, 408, 171

Nazé, Y., Rauw, G., Manfroid, J., Chu, Y.-H., \& Vreux, J.-M. 2003b, A\&A, 401, L13

Niemela, V. S., Heathcote, S. R., \& Weller, W. G. 1991, IAUS, 143, 425

Ouchi, M., Shimasaku, K., Akiyama, M., et al. 2008, ApJS, 176, 301

Pakull, M. W. 1991, IAUS, 143, 391

Pakull, M. W. 2009, IAUS, 256, 437

Pakull, M. W., \& Angebault, L. P. 1986, Nature, 322, 511

Pakull, M. W., \& Motch, C. 1989, Nature, 337, 337

Pakull, M. W., Soria, R., \& Motch, C. 2010, Nature, 466, 209
Peimbert, M. 1990, Rep. Prog. Phys., 53, 1559

Peimbert, M., \& Costero, R. 1969, Boletin de los Observatorios Tonantzintla y Tacubaya, 5, 3

Pérez-Montero, E., \& Díaz, A. I. 2005, MNRAS, 361, 1063

Pettini, M., \& Pagel, B. E. J. 2004, MNRAS, 348, L59

Pilyugin, L. S., Vílchez, J. M., \& Thuan, T. X. 2006, MNRAS, 370, 1928

Polcaro, V. F., Rossi, C., Norci, L., \& Viotti, R. 1995, A\&A, 303, 211

Rosolowsky, E., \& Simon, J. D. 2008, ApJ, 675, 1213

Rühling, U. 2008, Diplomarbeit, Universität Potsdam, Germany http: //www .astro.physik. uni-potsdam.de/ftp/ruehling/ Diplomarbeit-Ruehling-2008.pdf

Russell, S. C., \& Dopita, M. A. 1990, ApJS, 74, 93

Schaerer, D. 1996, ApJ, 467, L17

Schaerer, D. 2002, A\&A, 382, 28

Schaerer, D. 2003, A\&A, 397, 527

Schaerer, D. 2008, IAU Symp., 255, 66

Schaerer, D., \& Maeder, A. 1992, A\&A, 263, 129

Schaerer, D., Contini, T., \& Pindao, M. 1999, A\&AS, 136, 35

Schmutz, W., Leitherer, C., \& Gruenwald, R. 1992, PASP, 104, 1164

Schmutz, W. 1993, Space Sci. Rev., 66, 253

Schmutz, W., \& De Marco, O. 1999, Wolf-Rayet Phenomena in Massive Stars and Starburst Galaxies, 193, 147

Shaw, R. A., \& Dufour, R. J. 1994, Astronomical Data Analysis Software and Systems III, 61, 327

Smith, L. F., Shara, M. M., \& Moffat, A. F. J. 1996, MNRAS, 281, 163

Smith, L. J., Norris, R. P. F., \& Crowther, P. A. 2002, MNRAS, 337, 1309

Tayal, S. S., \& Gupta, G. P. 1999, ApJ, 526, 544

Testor, G., \& Pakull, M. 1989, Recent Developments of Magellanic Cloud Research, 141

Thuan, T. X., \& Izotov, Y. I. 2005, ApJS, 161, 240

Vink, J. S., \& de Koter, A. 2005, A\&A, 442, 587

Walborn, N. R., Drissen, L., Parker, J. Wm., et al. 1999, AJ, 118, 1684

Williams, J. P., Bergin, E. A., Caselli, P., Myers, P. C., \& Plume, R. 1998, ApJ, 503, 689

Zaritsky, D., Elston, R., \& Hill, J. M. 1989, AJ, 97, 97 